\title{
Finite element approximation of a Cahn-Hilliard-Navier-Stokes system
}

\author{
DAVID KAY ${ }^{\dagger}$ \\ Oxford University Computing Laboratory, Oxford, OX1 3QD, U.K. \\ VANESSA STYLES ${ }^{\ddagger}$ AND RICHARD WELFORD ${ }^{\S}$ \\ Department of Mathematics, University of Sussex, Brighton, BN1 9RF, U.K.
}

[Received 1 June 2006 and in revised form 20 June 2007]

\begin{abstract}
We consider a semi-discrete and a practical fully discrete finite element approximation of a CahnHilliard-Navier-Stokes system. This system arises in the modelling of multiphase fluid systems. We show order $h$ error estimate between the solution of the system and the solution of the semidiscrete approximation. We also show the convergence of the fully discrete approximation. Finally, we present an efficient implementation of the fully discrete scheme together with some numerical simulations.
\end{abstract}

\section{Introduction}

The Cahn-Hilliard equation [10, 11] is a phenomenological model of phase transitions (see the survey work of Elliott [15] for details). Coupling the Cahn-Hilliard and Navier-Stokes equations yields a model for the dynamics of multiphase fluids that is used to model phenomena such as hydrodynamic effects during spinodal decomposition and the behaviour of polymer fluids (see for example [27, 26]). The Cahn-Hilliard-Navier-Stokes system that we consider is known as 'Model H' in the nomenclature of Hohenberg and Halperin [22]:

(R) Find $\{c(x, t), w(x, t), \mathbf{u}(x, t), p(x, t)\}$ such that

$$
\begin{array}{cl}
\partial_{t} c-\frac{1}{\mathrm{Pe}} \nabla \cdot(b(c) \nabla w)+\mathbf{u} \cdot \nabla c=0 & \text { in } \Omega_{T}:=\Omega \times(0, T), \\
w=\Phi^{\prime}(c)-\gamma^{2} \Delta c & \text { in } \Omega_{T}, \\
\partial_{t} \mathbf{u}-\frac{1}{\operatorname{Re}} \Delta \mathbf{u}+\mathbf{u} \cdot \nabla \mathbf{u}+\nabla p+K c \nabla w=0 & \text { in } \Omega_{T}, \\
\nabla \cdot \mathbf{u}=0 & \text { in } \Omega_{T}, \\
c(x, 0)=c_{0}(x), \quad \mathbf{u}(x, 0)=\mathbf{u}_{0}(x) & \forall x \in \Omega, \\
\partial_{\mathbf{n}} c=\partial_{\mathbf{n}} w=0, \quad \mathbf{u}=\mathbf{g} & \text { on } \partial \Omega \times(0, T),
\end{array}
$$

where $\mathbf{g}$ satisfies $\mathbf{g} \cdot \mathbf{n}=0$ on $\partial \Omega$. Here $\Omega$ is a bounded convex polygonal domain in $\mathbb{R}^{2}$, with boundary $\partial \Omega$ that has outward pointing unit normal $\mathbf{n}$. The concentration order parameter $c$ is such that $c(x, t) \approx 1$ (respectively $c(x, t) \approx-1$ ) if and only if at time $t$ fluid 1 (respectively fluid 2 ) is

\footnotetext{
†E-mail: david.kay@comlab.ox.ac.uk

E-mail: v.styles@sussex.ac.uk

${ }^{\S}$ Supported by EPSRC grant GR/R8951/01. E-mail: r.r.welford@ sussex.ac.uk
} 
present at the point $x$. The mean velocity field $\mathbf{u}$ is defined to be

$$
\mathbf{u}=\frac{1+c}{2} \mathbf{u}_{1}+\frac{1-c}{2} \mathbf{u}_{2}
$$

where $\mathbf{u}_{i}$ is the velocity field of fluid component $i$. The chemical potential and the pressure in the system are defined by $w$ and $p$ respectively, and the Péclet number Pe, the Reynolds number Re and the capillary number $K$ are given constants. The interface parameter $\gamma>0$ is also a given constant that is assumed to be small. We take the mobility function $b(\cdot)$ in $(1.1 \mathrm{a})$ to be of the form

$$
\exists b_{1}, b_{2}>0 \quad b_{1} \leqslant b(x) \leqslant b_{2} \quad \forall x \in \Omega
$$

and we take the free energy $\Phi(\cdot)$ in $1.1 \mathrm{~b}$ to be

$$
\Phi(c)=\frac{1}{4}\left(1-c^{2}\right)^{2} .
$$

By $\partial_{t} \eta$ we mean $\partial \eta / \partial t$ and similarly for $\partial_{\mathbf{n}} \eta$.

In many fluid flow applications more than one fluid is present, with the components often separated by an interfacial region of partial miscibility, even if the fluids themselves are immiscible. Such multicomponent systems can use either a sharp or a diffuse interface to model these free boundaries, but in the evolution of such problems often breakup or coalescence of interfaces can occur and it is important that the model used can accurately capture this change of topology. Phase field models, such as the Cahn-Hilliard equation, place a diffuse interface between the phases of the system, which allows for a natural description of topological changes in the model without relying on any additional input from the user. The numerical simulation of these models is an area of research currently undergoing intense study (see for example [2, 9, 23, 26, 27]), with the majority of the work so far concentrating on finite difference discretisations. However, despite there being much work on finite element discretizations for both the Cahn-Hilliard (see for example [3, 4, 6]) and the Navier-Stokes equations (see for example [21]) separately, there has been little work on finite element discretizations for Cahn-Hilliard-Navier-Stokes problems.

Adaptive finite element techniques are well suited to phase field modelling since the solution of such models rapidly varies over the interfacial regions while away from the interface, in the bulk regions, it is close to \pm 1 . This means that it is natural to assume that most of the computational work is needed in the interfacial region. Additionally this region needs to be accurately resolved, as otherwise spurious numerical solutions can occur (see [18]). As a result it is common to couple phase field modelling with adaptive mesh refinement whereby the mesh is locally refined close to and inside of the interfacial region and coarsened elsewhere (see Section 5).

The model $(\mathbf{R})$ gives a diffuse interface description of binary incompressible fluid flow (see [1]). An existence/uniqueness result for (R), with (1.4) replaced by a more general form for $\Phi(\cdot)$, is given in [12], and in [7] an existence result for the problem with degenerate mobility is presented. The motivation behind this paper is to analyse semi-discrete and fully discrete finite element discretizations of $(\mathbf{R})$ and to present some numerical computations. In Section 2 we introduce notation and define a weak formulation, $(\mathbf{P})$, of $(\mathbf{R})$; then in Section 3 we introduce a continuous in time finite element approximation, $\left(\mathbf{P}_{h}\right)$, of $(\mathbf{P})$ and we use the techniques presented in [16] to show order $h$ error estimate between the solutions of $(\mathbf{P})$ and $\left(\mathbf{P}_{h}\right)$. In Section 4 we introduce a practical discrete in time and space finite element approximation, $\left(\mathbf{P}_{h, \tau}\right)$, of $(\mathbf{P})$ that we show converges to $(\mathbf{P})$ as the spatial and temporal parameters tend to zero. We conclude with Section 5 in which we present an efficient implementation of the practical fully discrete scheme together with some numerical results. 
Remark 1.1 1. Setting $\mathbf{u} \equiv \mathbf{0}$ in $(\mathbf{R})$ yields the Cahn-Hilliard equation, while setting $c \equiv 0$ in $(\mathbf{R})$ yields the Navier-Stokes equation.

2. The term $c \nabla w$ in the Navier-Stokes equation (1.1c can be replaced by $w \nabla c$ where the additional term $\nabla(w c)$ is absorbed into the pressure term.

REMARK 1.2 Upon completion of this work we became aware of a related paper by Feng [19]. In this paper a fully discrete finite element approximation for a Cahn-Hilliard-Navier-Stokes system in three space dimensions is developed and analysed. In particular the author shows the convergence of the numerical scheme and establishes the sharp interface limit of the model by utilising a discrete energy law. The main differences between the convergence proof in [19] and the one in Section 4 of this paper is that the finite element discretization in [19] is fully implicit, resulting in a CahnHilliard-Navier-Stokes coupled system. In this paper we analyse a semi-implicit scheme that only requires the solutions of separate Cahn-Hilliard and Navier-Stokes equations. As a result we require different techniques to obtain the stability bounds on the approximate solutions.

\section{Notation and auxiliary results}

Let $L^{p}(\Omega)$ denote the space of $p$-integrable functions with norm denoted by $\|\cdot\|_{0, p}$, where for simplicity of notation we set $\|\cdot\|_{0,2}=\|\cdot\|$. Furthermore let $W^{m, p}(\Omega)$ and $H^{m}(\Omega)$ be the usual Sobolev spaces with norms $\|\cdot\|_{m, p}$ and $\|\cdot\|_{m}$ respectively and let $\left(H^{1}(\Omega)\right)^{\prime}$ denote the dual space of $H^{1}(\Omega)$. Let $H_{0}^{1}(\Omega)$ be defined by

$$
H_{0}^{1}(\Omega):=\left\{\eta \in H^{1}(\Omega): \eta=0 \text { on } \partial \Omega\right\},
$$

and let $H^{-1}(\Omega)$ denote the dual space of $H_{0}^{1}(\Omega)$ with norm $\|\cdot\|_{-1}$. Let $\mathbf{L}^{p}(\Omega)=\left(L^{p}(\Omega)\right)^{2}$ and $\mathbf{H}^{m}(\Omega):=\left(H^{m}(\Omega)\right)^{2}$ for $m=-1,1$ and 2 . We define the following spaces:

$$
\begin{aligned}
V & =\left\{v \in H^{1}(\Omega):(v, 1)=0\right\}, \\
\mathcal{F} & =\left\{v \in\left(H^{1}(\Omega)\right)^{\prime}:\langle v, 1\rangle=0\right\}, \\
\mathbf{W} & =\left\{\mathbf{v} \in \mathbf{H}^{1}(\Omega):(\nabla \cdot \mathbf{v}, \eta)=0 \forall \eta \in L^{2}(\Omega)\right\}, \\
\mathbf{W}_{0} & =\{\mathbf{v} \in \mathbf{W}: \mathbf{v}=\mathbf{0} \text { on } \partial \Omega\},
\end{aligned}
$$

where $(\cdot, \cdot)$ denotes the $L^{2}$ inner product and $\langle\cdot, \cdot\rangle$ denotes the duality pairing between $\left(H^{1}(\Omega)\right)^{\prime}$ and $H^{1}(\Omega)$.

For $\mathbf{v} \in \mathbf{H}^{1}(\Omega), \eta, \xi \in H^{1}(\Omega)$ we define

$$
\mathbf{a}(\eta, \xi, \mathbf{v}):=\int_{\Omega}[\eta \nabla \xi \cdot \mathbf{v}] \mathrm{d} x,
$$

and for $\mathbf{u}, \mathbf{v}, \mathbf{w} \in \mathbf{H}^{1}(\Omega)$ we define

$$
B(\mathbf{u}, \mathbf{v}, \mathbf{w}):=\frac{1}{2} \int_{\Omega}[(\mathbf{u} \cdot \nabla) \mathbf{v} \cdot \mathbf{w}] \mathrm{d} x-\frac{1}{2} \int_{\Omega}[(\mathbf{u} \cdot \nabla) \mathbf{w} \cdot \mathbf{v}] \mathrm{d} x .
$$

Finally, for $\mathbf{u}, \mathbf{v}, \mathbf{w} \in \mathbf{H}^{1}(\Omega)$ we define

$$
(\nabla \mathbf{v}: \nabla \mathbf{w}):=\int_{\Omega} \nabla \mathbf{v}: \nabla \mathbf{w} \mathrm{d} x
$$

where the product $\mathcal{E}: \mathcal{F}$ of two $n \times n$ matrices $\mathcal{E}$ and $\mathcal{F}$ is defined to be $\sum_{i, j=1}^{n} \mathcal{E}_{i j} \mathcal{F}_{i j}$. 
REMARK 2.1 The trilinear form $B(\mathbf{u}, \mathbf{v}, \mathbf{w})$ in 2.3 that is associated with the nonlinearity in the Navier-Stokes equation has the following property:

$$
B(\mathbf{v}, \mathbf{u}, \mathbf{u})=0 .
$$

Next we introduce the Green's operator $\mathcal{G}: \mathcal{F} \rightarrow V$ such that

$$
(\nabla[\mathcal{G} z], \nabla \eta)=\langle z, \eta\rangle \quad \forall \eta \in H^{1}(\Omega) .
$$

The well-posedness of $\mathcal{G}$ follows from the generalised Lax-Milgram theorem and the Poincaré inequality

$$
\|\eta\| \leqslant C(\|\nabla \eta\|+|(\eta, 1)|) \quad \forall \eta \in H^{1}(\Omega) .
$$

Let $X, Y, Z$ be Banach spaces with a compact embedding $X \hookrightarrow Y$ and a continuous embedding $Y \hookrightarrow Z$. Then the embeddings

$$
\left\{\eta \in L^{2}(0, T ; X): \partial_{t} \eta \in L^{2}(0, T ; Z)\right\} \hookrightarrow L^{2}(0, T ; Y)
$$

and

$$
\left\{\eta \in L^{\infty}(0, T ; X): \partial_{t} \eta \in L^{2}(0, T ; Z)\right\} \hookrightarrow C([0, T] ; Y)
$$

are compact. In the following we often use the well-known result

$$
\|\eta\|_{0, r} \leqslant C\|\eta\|_{1} \quad \forall \eta \in H^{1}(\Omega) \text { and } r \in[2, \infty),
$$

in particular when bounding the triple

$$
\mathbf{a}(\eta, \chi, \boldsymbol{\xi})=\left|\int_{\Omega} \eta \nabla \chi \cdot \boldsymbol{\xi} \mathrm{d} x\right| \leqslant\|\eta \boldsymbol{\xi}\|\|\nabla \chi\| \leqslant\|\eta\|_{0,4}\|\boldsymbol{\xi}\|_{0,4}\|\nabla \chi\| \leqslant\|\eta\|_{1}\|\boldsymbol{\xi}\|_{1}\|\nabla \chi\| .
$$

Furthermore we note that for $\mu=1-2 / r$ and $\sigma=1 / 2-2 / r$ we have

$$
\|\eta\|_{0, r} \leqslant C\|\eta\|^{1-\mu}\|\eta\|_{1}^{\mu} \quad \forall \eta \in H^{1}(\Omega) \text { and } r \in[2, \infty)
$$

and

$$
\|\eta\|_{0, r} \leqslant C\|\eta\|_{0,4}^{1-\sigma}\|\eta\|_{1,4}^{\sigma} \quad \forall \eta \in W^{1,4}(\Omega) \text { and } r \in[4, \infty] .
$$

Throughout this work $C, C_{1}$ and $C_{2}$ will denote constants whose value may change from line to line. Furthermore $C_{1}$ will denote a constant that can be taken to be arbitrarily small.

\subsection{A weak formulation of the problem}

We now introduce a weak formulation of $(1.1 \mathrm{a}-\mathrm{f})$ :

Find $\{c(x, t), w(x, t), \mathbf{u}(x, t), p(x, t)\}$ such that

$$
\begin{array}{clc}
\left(\partial_{t} c, \eta\right)+\frac{1}{\operatorname{Pe}}(b(c) \nabla w, \nabla \eta)=\mathbf{a}(c, \eta, \mathbf{u}) & \forall \eta \in H^{1}(\Omega), & \text { (2.11a) } \\
(w, \eta)=\left(\Phi^{\prime}(c), \eta\right)+\gamma^{2}(\nabla c, \nabla \eta) & \forall \eta \in H^{1}(\Omega), & \text { (2.11b) } \\
\left(\partial_{t} \mathbf{u}, \mathbf{v}\right)+\frac{1}{\operatorname{Re}}(\nabla \mathbf{u}: \nabla \mathbf{v})+B(\mathbf{u}, \mathbf{u}, \mathbf{v})-(p, \nabla \cdot \mathbf{v})+K \mathbf{a}(c, w, \mathbf{v})=0 & \forall \mathbf{v} \in \mathbf{H}_{0}^{1}(\Omega), & \text { (2.11c) } \\
(\nabla \cdot \mathbf{u}, \chi)=0 & \forall \chi \in L^{2}(\Omega), & \text { (2.11d) } \\
c(x, 0)=c_{0}(x) \in H^{2}(\Omega) \cap V, \quad \mathbf{u}(x, 0)=\mathbf{u}_{0}(x) \in \mathbf{W}_{0} \cap \mathbf{H}^{2}(\Omega) & \forall x \in \Omega, & \text { (2.11e) } \\
\mathbf{u}=\mathbf{g} & \text { on } \partial \Omega \times(0, T) . & \text { (2.11f) }
\end{array}
$$


In Sections 3 and 4 we analyse the following weak formulation of (2.11a-f) with $\mathbf{g}=\mathbf{0}$ in which for simplicity of presentation we have set $b(\cdot) \equiv 1$ and $\mathrm{Pe}=\mathrm{Re}=K=1$ :

(P) Find $\{c(x, t), w(x, t), \mathbf{u}(x, t)\} \in V \times H^{1}(\Omega) \times \mathbf{W}_{0}$ such that

$$
\begin{array}{cl}
\left(\partial_{t} c, \eta\right)+(\nabla w, \nabla \eta)=\mathbf{a}(c, \eta, \mathbf{u}) & \forall \eta \in H^{1}(\Omega), \\
(w, \eta)=\left(\Phi^{\prime}(c), \eta\right)+\gamma^{2}(\nabla c, \nabla \eta) & \forall \eta \in H^{1}(\Omega), \\
\left(\partial_{t} \mathbf{u}, \mathbf{v}\right)+(\nabla \mathbf{u}: \nabla \mathbf{v})+B(\mathbf{u}, \mathbf{u}, \mathbf{v})+\mathbf{a}(c, w, \mathbf{v})=0 & \forall \mathbf{v} \in \mathbf{W}_{0}, \\
c(x, 0)=c_{0}(x) \in H^{2}(\Omega) \cap V, \quad \mathbf{u}(x, 0)=\mathbf{u}_{0}(x) \in \mathbf{W}_{0} \cap \mathbf{H}^{2}(\Omega) & \forall x \in \Omega .
\end{array}
$$

REMARK 2.2 We note that $2.12 \mathrm{c}$ ) can also be written as

$$
\left(\partial_{t} \mathbf{u}, \mathbf{v}\right)+(\nabla \mathbf{u}: \nabla \mathbf{v})+B(\mathbf{u}, \mathbf{u}, \mathbf{v})-(p, \nabla \cdot \mathbf{v})+\mathbf{a}(c, w, \mathbf{v})=0 \quad \forall \mathbf{v} \in \mathbf{H}_{0}^{1}(\Omega) .
$$

Since $\mathbf{g}=\mathbf{0}$, from [12] and [30] it follows that there exists a unique solution $\{c, w, \mathbf{u}\}$ of $(\mathbf{P})$ that satisfies

$$
\left\{\begin{array}{l}
c \in L^{\infty}\left(0, T ; H^{2}(\Omega) \cap V\right) \cap H^{1}\left(0, T ; L^{2}(\Omega)\right), \\
\mathbf{u} \in L^{\infty}\left(0, T ; \mathbf{W}_{0} \cap \mathbf{H}^{2}(\Omega)\right) \cap H^{1}\left(0, T ; \mathbf{L}^{2}(\Omega)\right), \\
w \in L^{\infty}\left(0, T ; L^{2}(\Omega)\right) \cap L^{2}\left(0, T ; H^{2}(\Omega)\right) \cap H^{1}\left(0, T ; L^{2}(\Omega)\right), \\
p \in L^{2}\left(0, T ; H^{1}(\Omega)\right) .
\end{array}\right.
$$

\subsection{Finite element notation}

In Sections 3 and 4 we consider a semi-discrete and a fully discrete finite element approximation of (P) under the following assumptions on the mesh.

Let $\mathcal{T}^{h}$ be a quasi-uniform partitioning of $\Omega$ into disjoint open simplices $\{\kappa\}$ with $h_{\kappa}:=\operatorname{diam}(\kappa)$ and $h:=\max _{\kappa \in \mathcal{T}^{h}} h_{\kappa}$, so that $\bar{\Omega}=\bigcup_{\kappa \in \mathcal{T}^{h}} \bar{\kappa}$.

Also we assume that $\mathcal{T}^{h}$ is weakly acute; that is, for any pair of adjacent triangles the sum of the opposite angles relative to the common side does not exceed $\pi$. We define $\mathcal{T}^{h / 2}$ to be the mesh obtained from $\mathcal{T}^{h}$ by refining each simplex $\kappa$ into four similar triangles by joining the midpoints of each edge of $\kappa$.

Associated with $\mathcal{T}^{h}$ and $\mathcal{T}^{h / 2}$ are the finite element spaces

$$
\begin{aligned}
S^{h} & :=\left\{v \in C(\bar{\Omega}):\left.v\right|_{\kappa} \text { is linear } \forall \kappa \in \mathcal{T}^{h}\right\} \subset H^{1}(\Omega), \\
S^{h / 2} & :=\left\{v \in C(\bar{\Omega}):\left.v\right|_{\kappa} \text { is linear } \forall \kappa \in \mathcal{T}^{h / 2}\right\} \subset H^{1}(\Omega) .
\end{aligned}
$$

Let $\pi^{h}: C(\bar{\Omega}) \rightarrow S^{h}$ be the piecewise linear Lagrange interpolation operator such that $\left(\pi^{h} v\right)\left(x_{j}\right)=v\left(x_{j}\right)$ for all $j=1, \ldots, J$, where $J$ denotes the number of nodes of $\mathcal{T}^{h}$. Similarly we define $\pi^{h}:(C(\Omega))^{2} \rightarrow\left(S^{h / 2}\right)^{2}$ and we note that for all $q \in(2, \infty]$,

$$
\begin{array}{rlrl}
\left\|\nabla\left(I-\boldsymbol{\pi}^{h}\right) \boldsymbol{\eta}\right\| & \leqslant C h\|\boldsymbol{\eta}\|_{2} & & \forall \boldsymbol{\eta} \in \mathbf{H}^{2}(\Omega), \\
\left\|\nabla\left(I-\boldsymbol{\pi}^{h}\right) \boldsymbol{\eta}\right\|_{0, q} \leqslant C\|\nabla \boldsymbol{\eta}\|_{0, q} & & \forall \boldsymbol{\eta} \in \mathbf{H}^{1, q}(\Omega) .
\end{array}
$$


We define the finite element spaces, analogous to the earlier continuous spaces,

$$
\begin{aligned}
V^{h} & =\left\{v \in S^{h}:(v, 1)=0\right\}, \\
\mathbf{W}^{h} & =\left\{\mathbf{v} \in\left(S^{h / 2}\right)^{2}:(\nabla \cdot \mathbf{v}, \chi)=0 \forall \chi \in S^{h}\right\}, \\
\mathbf{W}_{0}^{h} & =\left\{\mathbf{v} \in \mathbf{W}^{h}: \mathbf{v}=\mathbf{0} \text { on } \partial \Omega\right\} .
\end{aligned}
$$

We define a discrete $L^{2}(\Omega)$ inner product on $C(\bar{\Omega})$ via

$$
(u, v)_{h}:=\int_{\Omega} \pi^{h}(u v) \mathrm{d} x .
$$

For $\eta, \chi \in C(\bar{\Omega})$ we set

$$
I_{h}(\eta, \chi):=(\eta, \chi)_{h}-(\eta, \chi)
$$

and we note that

$$
\left|I_{h}(\eta, \chi)\right|=\left|(\eta, \chi)_{h}-(\eta, \chi)\right| \leqslant C h\|\eta\|\|\nabla \chi\| \quad \forall \eta, \chi \in S^{h} .
$$

It is well known that the discrete inner product 2.16 induces a norm on $S^{h} \subset C(\bar{\Omega})$, via

$$
\|\chi\|_{h}^{2}:=(\chi, \chi)_{h} \quad \forall \chi \in S^{h}
$$

and that there is an equivalence of norms between $\|\cdot\|$ and $\|\cdot\|_{h}$, i.e.

$$
C\|\chi\| \leqslant\|\chi\|_{h} \leqslant C\|\chi\|, \quad \forall \chi \in S^{h} .
$$

The Poincaré inequality 2.6 together with 2.18 -2.20 yields, for $h$ sufficiently small, the following discrete Poincaré inequality:

$$
\|\chi\|_{h} \leqslant C\left(\|\nabla \chi\|+\left|(\chi, 1)_{h}\right|\right) \quad \forall \chi \in S^{h},
$$

so

$$
\|\chi\|_{1} \leqslant C\|\nabla \chi\| \quad \forall \chi \in V^{h} .
$$

We introduce the $L^{2}$ projection $Q^{h}: L^{2}(\Omega) \rightarrow S^{h}$ defined by

$$
\left(Q^{h} \eta, \chi\right)_{h}=(\eta, \chi) \quad \forall \chi \in S^{h}
$$

and we note that

$$
\left\|\left(I-Q^{h}\right) \eta\right\|+h\left\|\nabla\left(I-Q^{h}\right) \eta\right\| \leqslant C h\|\nabla \eta\| \quad \forall \eta \in H^{1}(\Omega) .
$$

We also introduce the projection $P^{h}$ on $S^{h}$, with respect to the inner product $(\nabla v, \nabla w)$, so that for $\eta \in H^{1}(\Omega)$,

$$
\left(\nabla P^{h} \eta, \nabla \chi\right)=(\nabla \eta, \nabla \chi) \quad \forall \chi \in S^{h} \quad \text { and } \quad\left(P^{h} \eta, 1\right)=(\eta, 1)
$$

and we note that

$$
\left\|P^{h} \eta-\eta\right\|+h\left\|\nabla\left(P^{h} \eta-\eta\right)\right\| \leqslant C h^{s}\|\eta\|_{s} \quad \forall \eta \in H^{s}(\Omega), s=1,2 .
$$


We also define $\mathbf{P}^{h}: \mathbf{W}_{0} \rightarrow \mathbf{W}_{0}^{h}$ to be the Stokes projection such that for all $\mathbf{v} \in \mathbf{W}_{0}$,

$$
\left(\nabla \mathbf{P}^{h} \mathbf{v}: \nabla \chi\right)=(\nabla \mathbf{v}: \nabla \chi) \quad \forall \chi \in \mathbf{W}_{0}^{h}
$$

and we note that

$$
\left\|\mathbf{P}^{h} \mathbf{v}-\mathbf{v}\right\|+h\left\|\nabla\left(\mathbf{P}^{h} \mathbf{v}-\mathbf{v}\right)\right\| \leqslant C h^{s}\|\mathbf{v}\|_{s} \quad \forall \mathbf{v} \in \mathbf{H}^{s}(\Omega) \cap \mathbf{W}_{0}, s=1,2 .
$$

We introduce the 'discrete Laplacian' operator $\Delta^{h}: S^{h} \rightarrow V^{h}$ such that

$$
\left(\Delta^{h} z_{h}, \chi\right)=-\left(\nabla z_{h}, \nabla \chi\right) \quad \forall \chi \in S^{h}
$$

and since we have a quasi-uniform family of partitionings and $\Omega$ is convex we have (see [5])

$$
\left\|\nabla z_{h}\right\|_{0, s} \leqslant C\left\|\Delta^{h} z_{h}\right\| \quad \forall s \in(1, \infty) .
$$

Next we introduce, similar to 2.5, the discrete Green's operators $\mathcal{G}^{h}: \mathcal{F} \rightarrow V^{h}$ and $\widehat{\mathcal{G}}^{h}$ : $V^{h} \rightarrow V^{h}$ such that

$$
\left(\nabla\left[\mathcal{G}^{h} \eta\right], \nabla \chi\right)=\langle\eta, \chi\rangle \quad \forall \chi \in S^{h}
$$

and

$$
\left(\nabla\left[\widehat{\mathcal{G}}^{h} z_{h}\right], \nabla \chi\right)=\left(z_{h}, \chi\right)_{h} \quad \forall \chi \in S^{h} .
$$

From [17] have

$$
\|\nabla \mathcal{G} v\| \leqslant C\left\|\nabla \mathcal{G}^{h} v\right\| \quad \forall v \in S^{h} .
$$

Finally we note the well-known inverse inequalities

$$
\begin{aligned}
\|\chi\|_{0,4} \leqslant C h^{-1 / 2}\|\chi\| & \forall \chi \in S^{h}, \\
\|\chi\|_{0, \infty} \leqslant C \sqrt{\ln (1 / h)}\|\chi\|_{1} & \forall \chi \in S^{h} .
\end{aligned}
$$

\section{Semi-discrete scheme}

In this section we consider a continuous in time finite element approximation, $\left(\mathbf{P}_{h}\right)$, of $(\mathbf{P})$ and we use the techniques presented in [16] to prove an error estimate between the solutions of $\left(\mathbf{P}_{h}\right)$ and $(\mathbf{P})$.

We consider the following semi-discrete approximation of $(\mathbf{P})$ :

$\left(\mathbf{P}_{h}\right)$ Find $\left\{c_{h}, w_{h}, \mathbf{u}_{h}\right\} \in V^{h} \times S^{h} \times \mathbf{W}_{0}^{h}$ such that

$$
\begin{array}{cc}
\left(\partial_{t} c_{h}, \chi\right)+\left(\nabla w_{h}, \nabla \chi\right)=\mathbf{a}\left(c_{h}, \chi, \mathbf{u}_{h}\right) & \forall \chi \in S^{h}, \\
\left(w_{h}, \chi\right)=\left(\Phi^{\prime}\left(c_{h}\right), \chi\right)+\gamma^{2}\left(\nabla c_{h}, \nabla \chi\right) & \forall \chi \in S^{h}, \\
\left(\partial_{t} \mathbf{u}_{h}, \chi\right)+\left(\nabla \mathbf{u}_{h}: \nabla \chi\right)+B\left(\mathbf{u}_{h}, \mathbf{u}_{h}, \chi\right)+\mathbf{a}\left(c_{h}, w_{h}, \chi\right)=0 & \forall \chi \in \mathbf{W}_{0}^{h}, \\
c_{h}(x, 0)=Q^{h}\left(c_{0}(x)\right) \in V^{h} \cap L^{\infty}(\Omega), \quad \mathbf{u}_{h}(x, 0)=\mathbf{P}^{h}\left(\mathbf{u}_{0}(x)\right) \in \mathbf{W}_{0}^{h} & \forall x \in \Omega .
\end{array}
$$

REMARK 3.1 From 3.1d, 2.24, 2.28) and 2.14 we have

$$
\left(\Phi\left(c_{h}(\cdot, 0)\right), 1\right)+\left\|c_{h}(\cdot, 0)\right\|_{1}+\left\|\mathbf{u}_{h}(\cdot, 0)\right\| \leqslant C .
$$


LEMMA 3.1 The system $\left(\mathbf{P}_{h}\right)$ satisfies the following stability estimates:

$$
\begin{gathered}
\left(\Phi\left(c_{h}(T)\right), 1\right)+\left\|c_{h}(T)\right\|_{1}^{2}+\left\|\mathbf{u}_{h}(T)\right\|^{2}+\int_{0}^{T}\left(\left\|\nabla \mathbf{u}_{h}\right\|^{2}+\left\|\nabla w_{h}\right\|^{2}\right) \mathrm{d} t \\
\leqslant C\left(\left(\Phi\left(c_{h}(0)\right), 1\right)+\left\|\nabla c_{h}(0)\right\|^{2}+\left\|\mathbf{u}_{h}(0)\right\|^{2}\right) \leqslant C, \\
\int_{0}^{T}\left\|c_{h}\right\|_{0, \infty}^{4} \mathrm{~d} t \leqslant C
\end{gathered}
$$

and

$$
\frac{1}{2}\left\|w_{h}(T)\right\|^{2}+\int_{0}^{T}\left\|\partial_{t} c_{h}\right\|^{2} \mathrm{~d} t \leqslant C(h)
$$

where $C(h)$ is a constant that depends on $h$.

Proof. Setting $\chi=w_{h}$ in 3.1a, $\chi=\partial_{t} c_{h}$ in 3.1b and $\chi=\mathbf{u}_{h}$ in 3.1c and combining the resulting equations gives

$$
\frac{\mathrm{d}}{\mathrm{d} t}\left(\Phi\left(c_{h}\right), 1\right)+\frac{\gamma^{2}}{2} \frac{\mathrm{d}}{\mathrm{d} t}\left\|\nabla c_{h}\right\|^{2}+\frac{1}{2} \frac{\mathrm{d}}{\mathrm{d} t}\left\|\mathbf{u}_{h}\right\|^{2}+\left\|\nabla \mathbf{u}_{h}\right\|^{2}+\left\|\nabla w_{h}\right\|^{2}+B\left(\mathbf{u}_{h}, \mathbf{u}_{h}, \mathbf{u}_{h}\right)=0
$$

and 3.3a follows by noting 2.4, integrating from 0 to $T$ and using 2.22) and 3.2).

To prove $3.3 \mathrm{~b}$ we take $\eta=\Delta^{h} c_{h}$ in $3.1 \mathrm{~b}$ and use 2.29a to obtain

$$
\begin{aligned}
\gamma^{2}\left\|\Delta^{h} c_{h}\right\|^{2} & =\left(\nabla w_{h}, \nabla c_{h}\right)+\left(\Phi^{\prime}\left(c_{h}\right), \Delta^{h} c_{h}\right) \\
& \leqslant \frac{1}{2}\left\|\nabla w_{h}\right\|^{2}+\frac{1}{2}\left\|\nabla c_{h}\right\|^{2}+C\left\|\Phi^{\prime}\left(c_{h}\right)\right\|^{2}+\frac{1}{2} \gamma^{2}\left\|\Delta^{h} c_{h}\right\|^{2} \\
& \leqslant \frac{1}{2}\left\|\nabla w_{h}\right\|^{2}+\frac{1}{2}\left\|\nabla c_{h}\right\|^{2}+C\left(\left\|c_{h}\right\|_{0,6}^{6}+\left\|c_{h}\right\|_{0,4}^{4}+\left\|c_{h}\right\|^{2}\right)+\frac{1}{2} \gamma^{2}\left\|\Delta^{h} c_{h}\right\|^{2} \\
& \leqslant \frac{1}{2}\left\|\nabla w_{h}\right\|^{2}+\frac{1}{2}\left\|\nabla c_{h}\right\|^{2}+C\left\|c_{h}\right\|_{1}^{6}+\frac{1}{2} \gamma^{2}\left\|\Delta^{h} c_{h}\right\|^{2} .
\end{aligned}
$$

Noting 2.29b and 2.22, integrating 3.5 from 0 to $T$ and using 3.3a we obtain

$$
\int_{0}^{T}\left\|\nabla c_{h}\right\|_{0,4}^{2} \mathrm{~d} t \leqslant C .
$$

From 3.6, 2.10 and 3.3a we conclude (3.3b).

To prove 3.4 we differentiate $3.1 \mathrm{~b}$ with respect to $t$ and then take $\chi=w_{h}$ in the resulting equation to obtain

$$
\frac{1}{2} \frac{\mathrm{d}}{\mathrm{d} t}\left\|w_{h}\right\|^{2}=\gamma^{2}\left(\partial_{t} \nabla c_{h}, \nabla w_{h}\right)+\left(\partial_{t}\left(\Phi^{\prime}\left(c_{h}\right)\right), w_{h}\right) .
$$

Next taking $\chi=\gamma^{2} \partial_{t} c_{h}$ in 3.1a), noting 1.4, 2.2 and combining the resulting equation with (3.7) we obtain

$$
\begin{aligned}
\frac{1}{2} \frac{\mathrm{d}}{\mathrm{d} t}\left\|w_{h}\right\|^{2}+\gamma^{2}\left\|\partial_{t} c_{h}\right\|^{2} \\
\quad=\left(\partial_{t}\left(\Phi^{\prime}\left(c_{h}\right)\right), w_{h}\right)+\gamma^{2} \mathbf{a}\left(c_{h}, \partial_{t} c_{h}, \mathbf{u}_{h}\right)=\left(\Phi^{\prime \prime}\left(c_{h}\right) \partial_{t} c_{h}, w_{h}\right)-\gamma^{2}\left(\nabla \cdot\left(c_{h} \mathbf{u}_{h}\right), \partial_{t} c_{h}\right) \\
\leqslant C\left\|\Phi^{\prime \prime}\left(c_{h}\right)\right\|_{0, \infty}^{2}\left\|w_{h}\right\|^{2}+\frac{1}{2} \gamma^{2}\left\|\partial_{t} c_{h}\right\|^{2}+C\left\|\nabla c_{h}\right\|_{0,4}^{2}\left\|\mathbf{u}_{h}\right\|_{0,4}^{2}+C\left\|\nabla \mathbf{u}_{h}\right\|^{2}\left\|c_{h}\right\|_{0, \infty}^{2} \\
\leqslant C\left(\left\|c_{h}\right\|_{0, \infty}^{4}+1\right)\left\|w_{h}\right\|^{2}+\frac{1}{2} \gamma^{2}\left\|\partial_{t} c_{h}\right\|^{2}+C\left\|\nabla c_{h}\right\|_{0,4}^{2}\left\|\mathbf{u}_{h}\right\|_{1}^{2}+C\left\|\nabla \mathbf{u}_{h}\right\|^{2}\left\|c_{h}\right\|_{0, \infty}^{2} .
\end{aligned}
$$


Using the above inequality and $2.32 \mathrm{a}$ ) we obtain

$$
\frac{1}{2} \frac{\mathrm{d}}{\mathrm{d} t}\left\|w_{h}\right\|^{2}+\frac{\gamma^{2}}{2}\left\|\partial_{t} c_{h}\right\|^{2} \leqslant \frac{C}{h}\left(\left(\left\|c_{h}\right\|_{0, \infty}^{4}+1\right)\left\|w_{h}\right\|^{2}+\left\|\nabla c_{h}\right\|^{2}\left\|\mathbf{u}_{h}\right\|_{1}^{2}+\left\|\nabla \mathbf{u}_{h}\right\|^{2}\left\|c_{h}\right\|_{1}^{2}\right)
$$

and 3.4 follows from Gronwall's inequality and 3.3a,b).

REMARK 3.1 From the theory of ordinary differential equations, noting (3.3a) and (3.4) it follows that the system $\left(\mathbf{P}_{h}\right)$ has a unique solution $\left\{c_{h}, w_{h}, \mathbf{u}_{h}\right\}$ on $[0, T]$ for all $T<\infty$.

Before we present the main result of this section we introduce some useful notation.

We define

$$
\begin{array}{lll}
E_{c}^{A}:=c-P^{h} c, & E_{c}^{h}:=P^{h} c-c_{h}, & E_{c}:=c-c_{h}=E_{c}^{A}+E_{c}^{h}, \\
E_{w}^{A}:=w-P^{h} w, & E_{w}^{h}:=P^{h} w-w_{h}, & E_{w}:=w-w_{h}=E_{w}^{A}+E_{w}^{h}, \\
E_{\mathbf{u}}^{A}:=\mathbf{u}-\mathbf{P}^{h} \mathbf{u}, & E_{\mathbf{u}}^{h}:=\mathbf{P}^{h} \mathbf{u}-\mathbf{u}_{h}, & E_{\mathbf{u}}:=\mathbf{u}-\mathbf{u}_{h}=E_{\mathbf{u}}^{A}+E_{\mathbf{u}}^{h} .
\end{array}
$$

From 2.26, 2.28, 23.8a-c) and 2.14) we have

$$
\left\|E_{c}^{A}\right\|_{1}+\left\|E_{\mathbf{u}}^{A}\right\|_{1} \leqslant C h \quad \text { and } \quad\left\|E_{w}^{A}\right\|_{1} \leqslant C h\|w\|_{2}
$$

and similarly we obtain

$$
\left\|\nabla \mathcal{G}^{h}\left(\partial_{t} E_{c}^{A}\right)\right\| \leqslant C h\left\|\partial_{t} c\right\| \quad \text { and } \quad\left\|\partial_{t} E_{\mathbf{u}}^{A}\right\|_{-1} \leqslant C h\left\|\partial_{t} \mathbf{u}\right\| .
$$

Using 3.8a, 3.9a and 2.22) we have

$$
\left\|E_{c}\right\|_{1} \leqslant\left\|E_{c}^{h}\right\|_{1}+\left\|E_{c}^{A}\right\|_{1} \leqslant\left\|E_{c}^{h}\right\|_{1}+C h \leqslant C\left\|\nabla E_{c}^{h}\right\|+C h,
$$

and similarly from 3.8c and 3.9a we have

$$
\left\|E_{\mathbf{u}}\right\|_{1} \leqslant\left\|E_{\mathbf{u}}^{h}\right\|_{1}+\left\|E_{\mathbf{u}}^{A}\right\|_{1} \leqslant\left\|E_{\mathbf{u}}^{h}\right\|_{1}+C h .
$$

We now proceed to bound the errors $E_{c}^{h}, E_{w}^{h}$ and $E_{\mathbf{u}}^{h}$. To this end we note the following lemma. LEMMA 3.2 We have

$$
\left|\left(\Phi^{\prime}\left(c_{h}\right)-\Phi^{\prime}(c), \partial_{t} E_{c}^{h}\right)\right| \leqslant \tilde{C}\left(h^{2}+\left\|\nabla E_{c}^{h}\right\|^{2}\right)+C_{1}\left\|\nabla \mathcal{G}^{h}\left(\partial_{t} E_{c}^{h}\right)\right\|^{2}
$$

where $\tilde{C}=C\left(\left\|c_{h}\right\|_{0, \infty}^{2}+1\right)^{2}$.

Proof. Noting 2.5 and 2.31 it follows that

$$
\begin{aligned}
\left|\left(\Phi^{\prime}(c)-\Phi^{\prime}\left(c_{h}\right), \partial_{t} E_{c}^{h}\right)\right| & =\left|\left(\nabla\left(\Phi^{\prime}(c)-\Phi^{\prime}\left(c_{h}\right)\right), \nabla \mathcal{G}\left(\partial_{t} E_{c}^{h}\right)\right)\right| \\
& \leqslant\left\|\nabla\left(\Phi^{\prime}(c)-\Phi^{\prime}\left(c_{h}\right)\right)\right\|\left\|\nabla \mathcal{G}\left(\partial_{t} E_{c}^{h}\right)\right\| \\
& \leqslant C\left\|\nabla\left(\Phi^{\prime}(c)-\Phi^{\prime}\left(c_{h}\right)\right)\right\|\left\|\nabla \mathcal{G}^{h}\left(\partial_{t} E_{c}^{h}\right)\right\| .
\end{aligned}
$$

From 2.14 and the Lipschitz continuity of $\Phi^{\prime \prime}$ we have

$$
\begin{aligned}
\left\|\nabla\left(\Phi^{\prime}(c)-\Phi^{\prime}\left(c_{h}\right)\right)\right\| & =\left\|\Phi^{\prime \prime}(c) \nabla c-\Phi^{\prime \prime}\left(c_{h}\right) \nabla c_{h}\right\| \\
& \leqslant\left\|\Phi^{\prime \prime}\left(c_{h}\right) \nabla\left(c-c_{h}\right)\right\|+\left\|\left(\Phi^{\prime \prime}(c)-\Phi^{\prime \prime}\left(c_{h}\right)\right) \nabla c\right\| \\
& \leqslant C\left(\left\|c_{h}\right\|_{0, \infty}^{2}+1\right)\left\|\nabla E_{c}\right\|+C\left(\left\|c_{h}\right\|_{0, \infty}+\|c\|_{0, \infty}\right)\left\|c-c_{h}\right\|_{0,4}\|\nabla c\|_{0,4} \\
& \leqslant C\left(\left\|c_{h}\right\|_{0, \infty}^{2}+1\right)\left(\left\|\nabla E_{c}\right\|+\left\|E_{c}\right\|_{1}\right) .
\end{aligned}
$$

The desired result follows from 3.12, 3.13, 3.3b and 3.10a). 
REMARK 3.2 We note from 2.28, 2.32a, 2.15b) and 2.14 that

$$
\begin{aligned}
\left\|\nabla \mathbf{P}^{h} \mathbf{u}\right\|_{0,4} & \leqslant\left\|\nabla\left(\pi^{h} \mathbf{u}-\mathbf{u}\right)\right\|_{0,4}+\left\|\nabla\left(\mathbf{P}^{h} \mathbf{u}-\pi^{h} \mathbf{u}\right)\right\|_{0,4}+\|\nabla \mathbf{u}\|_{0,4} \\
& \leqslant C\|\nabla \mathbf{u}\|_{0,4}+C h^{-1 / 2}\left\|\nabla\left(\mathbf{P}^{h} \mathbf{u}-\pi^{h} \mathbf{u}\right)\right\|+\|\nabla \mathbf{u}\|_{0,4} \\
& \leqslant C\|\nabla \mathbf{u}\|_{0,4}+C h^{1 / 2}\|\mathbf{u}\|_{2} \leqslant C .
\end{aligned}
$$

Similarly using 2.32b) we have

$$
\begin{aligned}
\left\|\mathbf{P}^{h} \mathbf{u}\right\|_{0, \infty} & \leqslant\left\|\boldsymbol{\pi}^{h} \mathbf{u}-\mathbf{u}\right\|_{0, \infty}+\left\|\mathbf{P}^{h} \mathbf{u}-\boldsymbol{\pi}^{h} \mathbf{u}\right\|_{0, \infty}+\|\mathbf{u}\|_{0, \infty} \\
& \leqslant C\|\mathbf{u}\|_{0, \infty}+C \sqrt{\ln (1 / h)}\left\|\mathbf{P}^{h} \mathbf{u}-\boldsymbol{\pi}^{h} \mathbf{u}\right\|_{1}+\|\mathbf{u}\|_{0, \infty} \\
& \leqslant C\|\mathbf{u}\|_{0, \infty}+C h \sqrt{\ln (1 / h)} \leqslant C .
\end{aligned}
$$

LEMMA 3.3 For almost every $t \in[0, T]$, we have

$$
\begin{aligned}
\frac{\gamma^{2}}{2} \frac{\mathrm{d}}{\mathrm{d} t}\left\|\nabla E_{c}^{h}\right\|^{2}+\frac{3}{4}\left\|\nabla E_{w}^{h}\right\|^{2}+\frac{1}{2} \frac{\mathrm{d}}{\mathrm{d} t}\left\|E_{\mathbf{u}}^{h}\right\|^{2} & +\frac{3}{4}\left\|\nabla E_{\mathbf{u}}^{h}\right\|^{2} \\
& \leqslant \widehat{C}\left(h^{2}+\left\|\nabla E_{c}^{h}\right\|^{2}+\left\|E_{\mathbf{u}}^{h}\right\|^{2}\right)+C_{1}\left\|\nabla \mathcal{G}^{h}\left(\partial_{t} E_{c}^{h}\right)\right\|^{2}
\end{aligned}
$$

where $\widehat{C}=C\left(1+\left\|c_{h}\right\|_{0, \infty}^{4}+\|w\|_{2}^{2}+\left\|\partial_{t} c\right\|^{2}+\left\|\partial_{t} \mathbf{u}\right\|^{2}\right)$.

Proof. From 2.12a, 2.25) and 3.8a) we have

$$
\left(\partial_{t} P^{h} c, \eta\right)+\left(\nabla P^{h} w, \nabla \eta\right)=\mathbf{a}(c, \eta, \mathbf{u})-\left(\partial_{t} E_{c}^{A}, \eta\right) \quad \forall \eta \in S^{h} .
$$

Setting $\chi=E_{w}^{h}$ in 3.1 a and subtracting the resulting equation from 3.16 with $\eta=E_{w}^{h}$ we obtain

$$
\left(\partial_{t} E_{c}^{h}, E_{w}^{h}\right)+\left\|\nabla E_{w}^{h}\right\|^{2}=\mathbf{a}\left(c, E_{w}^{h}, \mathbf{u}\right)-\mathbf{a}\left(c_{h}, E_{w}^{h}, \mathbf{u}_{h}\right)-\left(\partial_{t} E_{c}^{A}, E_{w}^{h}\right) .
$$

Similarly setting $\eta=\partial_{t} c_{h}-\partial_{t} P^{h} c=\partial_{t} E_{c}^{h}$ in 2.12b and noting (2.25) and $3.8 \mathrm{~b}$ we obtain

$$
\left(P^{h} w, \partial_{t} E_{c}^{h}\right)=\left(\Phi^{\prime}(c), \partial_{t} E_{c}^{h}\right)+\gamma^{2}\left(\nabla P^{h} c, \nabla \partial_{t} E_{c}^{h}\right)-\left(E_{w}^{A}, \partial_{t} E_{c}^{h}\right) .
$$

Setting $\chi=\partial_{t} E_{c}^{h}$ in $3.1 \mathrm{~b}$ and subtracting the resulting equation from 3.18 we obtain

$$
\left(E_{w}^{h}, \partial_{t} E_{c}^{h}\right)=\left(\Phi^{\prime}(c)-\Phi^{\prime}\left(c_{h}\right), \partial_{t} E_{c}^{h}\right)+\frac{\gamma^{2}}{2} \frac{\mathrm{d}}{\mathrm{d} t}\left\|\nabla E_{c}^{h}\right\|^{2}-\left(E_{w}^{A}, \partial_{t} E_{c}^{h}\right) .
$$

Combining (3.17) and (3.19) we obtain

$$
\begin{aligned}
\frac{\gamma^{2}}{2} \frac{\mathrm{d}}{\mathrm{d} t}\left\|\nabla E_{c}^{h}\right\|^{2}+\left\|\nabla E_{w}^{h}\right\|^{2}= & \mathbf{a}\left(c, E_{w}^{h}, \mathbf{u}\right)-\mathbf{a}\left(c_{h}, E_{w}^{h}, \mathbf{u}_{h}\right)-\left(\partial_{t} E_{c}^{A}, E_{w}^{h}\right) \\
& +\left(\Phi^{\prime}\left(c_{h}\right)-\Phi^{\prime}(c)+E_{w}^{A}, \partial_{t} E_{c}^{h}\right) .
\end{aligned}
$$


Next setting $\mathbf{v}=\mathbf{P}^{h} \mathbf{u}-\mathbf{u}_{h}=E_{\mathbf{u}}^{h}$ in 2.13) and noting 2.27) and 3.8c) gives

$$
\left(\partial_{t} \mathbf{P}^{h} \mathbf{u}, E_{\mathbf{u}}^{h}\right)+\left(\nabla \mathbf{P}^{h} \mathbf{u}: \nabla E_{\mathbf{u}}^{h}\right)=\left(p, \nabla \cdot E_{\mathbf{u}}^{h}\right)-\mathbf{a}\left(c, w, E_{\mathbf{u}}^{h}\right)-B\left(\mathbf{u}, \mathbf{u}, E_{\mathbf{u}}^{h}\right)-\left(\partial_{t} E_{\mathbf{u}}^{A}, E_{\mathbf{u}}^{h}\right) .
$$

Since $\mathbf{P}^{h} \mathbf{u}-\mathbf{u}_{h} \in \mathbf{W}_{0}^{h}$ it follows that

$$
\left(\nabla \cdot E_{\mathbf{u}}^{h}, q_{h}\right)=\left(\nabla \cdot\left(\mathbf{P}^{h} \mathbf{u}-\mathbf{u}_{h}\right), q_{h}\right)=0 \quad \forall q_{h} \in S^{h} .
$$

Setting $\chi=\mathbf{P}^{h} \mathbf{u}-\mathbf{u}_{h}=E_{\mathbf{u}}^{h}$ in 3.1c , subtracting the resulting equation from 3.21 and using 3.22) with $q_{h}=Q^{h} p$ we obtain

$$
\begin{aligned}
\frac{1}{2} \frac{\mathrm{d}}{\mathrm{d} t}\left\|E_{\mathbf{u}}^{h}\right\|^{2}+\left\|\nabla E_{\mathbf{u}}^{h}\right\|^{2}= & \mathbf{a}\left(c_{h}, w_{h}, E_{\mathbf{u}}^{h}\right)-\mathbf{a}\left(c, w, E_{\mathbf{u}}^{h}\right)+B\left(\mathbf{u}_{h}, \mathbf{u}_{h}, E_{\mathbf{u}}^{h}\right)-B\left(\mathbf{u}, \mathbf{u}, E_{\mathbf{u}}^{h}\right) \\
& -\left(\partial_{t} E_{\mathbf{u}}^{A}, E_{\mathbf{u}}^{h}\right)+\left(\nabla \cdot E_{\mathbf{u}}^{h}, p-Q^{h} p\right) .
\end{aligned}
$$

Next we note from $3.8 \mathrm{a}-\mathrm{c})$ that

$$
\begin{aligned}
\mathbf{a}\left(c, E_{w}^{h}, \mathbf{u}\right)-\mathbf{a}( & \left.c_{h}, E_{w}^{h}, \mathbf{u}_{h}\right)+\mathbf{a}\left(c_{h}, w_{h}, E_{\mathbf{u}}^{h}\right)-\mathbf{a}\left(c, w, E_{\mathbf{u}}^{h}\right) \\
& =\mathbf{a}\left(E_{c}, E_{w}^{h}, \mathbf{u}\right)+\mathbf{a}\left(c_{h}, E_{w}^{h}, E_{\mathbf{u}}\right)-\mathbf{a}\left(c_{h}, E_{w}, E_{\mathbf{u}}^{h}\right)-\mathbf{a}\left(E_{c}, w, E_{\mathbf{u}}^{h}\right) \\
& =\mathbf{a}\left(E_{c}, E_{w}^{h}, \mathbf{u}\right)-\mathbf{a}\left(E_{c}, w, E_{\mathbf{u}}^{h}\right)-\mathbf{a}\left(c_{h}, E_{w}^{A}, E_{\mathbf{u}}^{h}\right)+\mathbf{a}\left(c_{h}, E_{w}^{h}, E_{\mathbf{u}}^{A}\right) .
\end{aligned}
$$

Combining (3.20, 3.23 , and (3.24) yields

$$
\begin{aligned}
\frac{\gamma^{2}}{2} \frac{\mathrm{d}}{\mathrm{d} t} \| \nabla & E_{c}^{h}\left\|^{2}+\right\| \nabla E_{w}^{h}\left\|^{2}+\frac{1}{2} \frac{\mathrm{d}}{\mathrm{d} t}\right\| E_{\mathbf{u}}^{h}\left\|^{2}+\right\| \nabla E_{\mathbf{u}}^{h} \|^{2} \\
= & \mathbf{a}\left(E_{c}, E_{w}^{h}, \mathbf{u}\right)-\mathbf{a}\left(E_{c}, w, E_{\mathbf{u}}^{h}\right)-\mathbf{a}\left(c_{h}, E_{w}^{A}, E_{\mathbf{u}}^{h}\right) \\
& +\mathbf{a}\left(c_{h}, E_{w}^{h}, E_{\mathbf{u}}^{A}\right)-\left(\partial_{t} E_{c}^{A}, E_{w}^{h}\right)+\left(E_{w}^{A}, \partial_{t} E_{c}^{h}\right)+\left(\nabla \cdot E_{\mathbf{u}}^{h}, p-Q^{h} p\right) \\
& +B\left(\mathbf{u}_{h}, \mathbf{u}_{h}, E_{\mathbf{u}}^{h}\right)-B\left(\mathbf{u}, \mathbf{u}, E_{\mathbf{u}}^{h}\right)-\left(\partial_{t} E_{\mathbf{u}}^{A}, E_{\mathbf{u}}^{h}\right)+\left(\Phi^{\prime}\left(c_{h}\right)-\Phi^{\prime}(c), \partial_{t} E_{c}^{h}\right) .
\end{aligned}
$$

We now bound the terms on the right hand side of 3.25). From 2.8, 2.14, 3.3a, 3.9a) and 3.10a we obtain

$$
\begin{aligned}
\mid \mathbf{a}\left(E_{c},\right. & \left.E_{w}^{h}, \mathbf{u}\right)|+| \mathbf{a}\left(E_{c}, w, E_{\mathbf{u}}^{h}\right)|+| \mathbf{a}\left(c_{h}, E_{w}^{A}, E_{\mathbf{u}}^{h}\right)|+| \mathbf{a}\left(c_{h}, E_{w}^{h}, E_{\mathbf{u}}^{A}\right) \mid \\
& \leqslant\left\|E_{c}\right\|_{1}\left(\|\mathbf{u}\|_{1}\left\|\nabla E_{w}^{h}\right\|+\|\nabla w\|\left\|E_{\mathbf{u}}^{h}\right\|_{1}\right)+\left\|c_{h}\right\|_{1}\left(\left\|E_{\mathbf{u}}^{h}\right\|_{1}\left\|\nabla E_{w}^{A}\right\|+\left\|E_{\mathbf{u}}^{A}\right\|_{1}\left\|\nabla E_{w}^{h}\right\|\right) \\
& \leqslant \widehat{C}\left\|E_{c}\right\|_{1}^{2}+C_{1}\left\|\nabla E_{w}^{h}\right\|^{2}+C_{1}\left\|E_{\mathbf{u}}^{h}\right\|_{1}^{2}+C\left\|\nabla E_{w}^{A}\right\|^{2}+C\left\|E_{\mathbf{u}}^{A}\right\|_{1}^{2} \\
& \leqslant \widehat{C}\left(h^{2}+\left\|\nabla E_{c}^{h}\right\|^{2}\right)+C_{1}\left(\left\|E_{\mathbf{u}}^{h}\right\|_{1}^{2}+\left\|\nabla E_{w}^{h}\right\|^{2}\right) .
\end{aligned}
$$

Futhermore noting 2.30a and (3.9ab) we have

$$
\begin{aligned}
\left|\left(\partial_{t} E_{c}^{A}, E_{w}^{h}\right)\right|+ & \left|\left(E_{w}^{A}, \partial_{t} E_{c}^{h}\right)\right|+\left|\left(\partial_{t} E_{\mathbf{u}}^{A}, E_{\mathbf{u}}^{h}\right)\right| \\
\leqslant & \left\|\nabla \mathcal{G}^{h}\left(\partial_{t} E_{c}^{A}\right)\right\|\left\|\nabla E_{w}^{h}\right\|+\left\|\nabla E_{w}^{A}\right\|\left\|\nabla \mathcal{G}^{h}\left(\partial_{t} E_{c}^{h}\right)\right\|+\left\|\partial_{t} E_{\mathbf{u}}^{A}\right\|_{-1}\left\|E_{\mathbf{u}}^{h}\right\|_{1} \\
\leqslant & C\left\|\nabla \mathcal{G}^{h}\left(\partial_{t} E_{c}^{A}\right)\right\|^{2}+C_{1}\left\|\nabla E_{w}^{h}\right\|^{2}+C\left\|\nabla E_{w}^{A}\right\|^{2} \\
& \quad+C_{1}\left\|\nabla \mathcal{G}^{h}\left(\partial_{t} E_{c}^{h}\right)\right\|^{2}+C\left\|\partial_{t} E_{\mathbf{u}}^{A}\right\|_{-1}^{2}+C_{1}\left\|E_{\mathbf{u}}^{h}\right\|_{1}^{2} \\
\leqslant & \widehat{C} h^{2}+C_{1}\left(\left\|\nabla E_{w}^{h}\right\|^{2}+\left\|\nabla \mathcal{G}^{h}\left(\partial_{t} E_{c}^{h}\right)\right\|^{2}+\left\|E_{\mathbf{u}}^{h}\right\|_{1}^{2}\right) .
\end{aligned}
$$


From Remark 3.2, (2.4), 2.14), (3.9a) and (3.10b) we have

$$
\begin{aligned}
\left|B\left(\mathbf{u}, \mathbf{u}, E_{\mathbf{u}}^{h}\right)-B\left(\mathbf{u}_{h}, \mathbf{u}_{h}, E_{\mathbf{u}}^{h}\right)\right|= & \left|B\left(\mathbf{u}, E_{\mathbf{u}}^{A}, E_{\mathbf{u}}^{h}\right)+B\left(E_{\mathbf{u}}, \mathbf{P}^{h} \mathbf{u}, E_{\mathbf{u}}^{h}\right)+B\left(\mathbf{u}_{h}, E_{\mathbf{u}}^{h}, E_{\mathbf{u}}^{h}\right)\right| \\
= & \left|B\left(\mathbf{u}, E_{\mathbf{u}}^{A}, E_{\mathbf{u}}^{h}\right)+B\left(E_{\mathbf{u}}, \mathbf{P}^{h} \mathbf{u}, E_{\mathbf{u}}^{h}\right)\right| \\
\leqslant & \|\mathbf{u}\|_{0, \infty}\left(\left\|\nabla E_{\mathbf{u}}^{A}\right\|\left\|E_{\mathbf{u}}^{h}\right\|+\left\|\nabla E_{\mathbf{u}}^{h}\right\|\left\|E_{\mathbf{u}}^{A}\right\|\right) \\
& +\left\|E_{\mathbf{u}}\right\|\left\|\nabla \mathbf{P}^{h} \mathbf{u}\right\|_{0,4}\left\|E_{\mathbf{u}}^{h}\right\|_{0,4}+\left\|\mathbf{P}^{h} \mathbf{u}\right\|_{0, \infty}\left\|\nabla E_{\mathbf{u}}^{h}\right\|\left\|E_{\mathbf{u}}\right\| \\
\leqslant & C_{1}\left\|E_{\mathbf{u}}^{h}\right\|_{1}^{2}+C\left\|E_{\mathbf{u}}^{A}\right\|_{1}^{2}+C\left\|E_{\mathbf{u}}\right\|^{2} \\
\leqslant & C h^{2}+C_{1}\left\|E_{\mathbf{u}}^{h}\right\|_{1}^{2}+C\left\|E_{\mathbf{u}}^{h}\right\|^{2} .
\end{aligned}
$$

Finally, from 2.24) and (2.14) we have

$$
\left|\left(\nabla \cdot E_{\mathbf{u}}^{h}, Q^{h} p-p\right)\right| \leqslant\left\|\nabla E_{\mathbf{u}}^{h}\right\|\left\|Q^{h} p-p\right\| \leqslant C h^{2}+C_{1}\left\|\nabla E_{\mathbf{u}}^{h}\right\|^{2} .
$$

Combining 3.25 - 3.29 and noting 3.11) we obtain

$$
\begin{aligned}
\frac{\gamma^{2}}{2} \frac{\mathrm{d}}{\mathrm{d} t}\left\|\nabla E_{c}^{h}\right\|^{2}+\frac{1}{2} \frac{\mathrm{d}}{\mathrm{d} t}\left\|E_{\mathbf{u}}^{h}\right\|^{2}+\left\|\nabla E_{\mathbf{u}}^{h}\right\|^{2} & +\left\|\nabla E_{w}^{h}\right\|^{2} \\
\leqslant & C_{1}\left(\left\|\nabla E_{\mathbf{u}}^{h}\right\|^{2}+\left\|\nabla E_{w}^{h}\right\|^{2}+\left\|\nabla \mathcal{G}^{h}\left(\partial_{t} E_{c}^{h}\right)\right\|^{2}\right) \\
& +\widehat{C}\left(h^{2}+\left\|\nabla E_{c}^{h}\right\|^{2}+\left\|E_{\mathbf{u}}^{h}\right\|^{2}\right) .
\end{aligned}
$$

Taking $C_{1}=1 / 4$ in 3.30 ) gives the desired result.

Lemma 3.4 For all $t \in[0, T]$ we have

$$
\left\|\nabla \mathcal{G}^{h}\left(\partial_{t} E_{c}^{h}\right)\right\|^{2} \leqslant \widehat{C} h^{2}+C_{2}\left(\left\|\nabla E_{w}^{h}\right\|^{2}+\left\|E_{\mathbf{u}}^{h}\right\|_{1}^{2}+\left\|\nabla E_{c}^{h}\right\|^{2}\right)
$$

where $\widehat{C}=C\left(1+\left\|c_{h}\right\|_{0, \infty}^{4}+\|w\|_{2}^{2}+\left\|\partial_{t} c\right\|^{2}+\left\|\partial_{t} \mathbf{u}\right\|^{2}\right)$.

Proof. Replacing $\eta$ with $\chi$ in (3.16), combining the resulting equation with 3.1a) and noting 2.30a and 3.8a we obtain

$$
\left(\nabla \mathcal{G}^{h}\left(\partial_{t} E_{c}^{h}\right), \nabla \chi\right)=\left(\partial_{t} E_{c}^{h}, \chi\right)=-\left(\nabla E_{w}^{h}, \nabla \chi\right)+\mathbf{a}(c, \chi, \mathbf{u})-\mathbf{a}\left(c_{h}, \chi, \mathbf{u}_{h}\right)-\left(\partial_{t} E_{c}^{A}, \chi\right),
$$

so

$$
\left(\nabla \mathcal{G}^{h}\left(\partial_{t} E_{c}^{h}\right), \nabla \chi\right)=-\left(\nabla E_{w}^{h}, \nabla \chi\right)+\mathbf{a}\left(E_{c}, \chi, \mathbf{u}\right)-\mathbf{a}\left(c_{h}, \chi, E_{\mathbf{u}}\right)-\left(\partial_{t} E_{c}^{A}, \chi\right) .
$$

From (3.31, 2.8), 3.9b), 2.14) and 3.3a) we have

$$
\begin{aligned}
\left(\nabla \mathcal{G}^{h}\left(\partial_{t} E_{c}^{h}\right), \nabla \chi\right) & \leqslant\left\|\nabla E_{w}^{h}\right\|\|\nabla \chi\|+\|\nabla \chi\|\left(\|\mathbf{u}\|_{0, \infty}\left\|E_{c}\right\|+\left\|c_{h}\right\|_{1}\left\|E_{\mathbf{u}}\right\|_{1}\right)+\left\|\nabla \mathcal{G}^{h}\left(\partial_{t} E_{c}^{A}\right)\right\|\|\nabla \chi\| \\
& \leqslant \widehat{C} h+C\left(\left\|\nabla E_{w}^{h}\right\|+\left\|E_{\mathbf{u}}\right\|_{1}+\left\|E_{c}\right\|\right)\|\nabla \chi\| .
\end{aligned}
$$

From 3.10a,b) we obtain the desired result.

From Lemmas 3.3 and 3.4 with $C_{1}$ chosen such that $4 C_{2} C_{1} \leqslant 1$ we obtain the following result.

LEMMA 3.5 For almost every $t \in[0, T]$, we have

$$
\frac{\gamma^{2}}{2} \frac{\mathrm{d}}{\mathrm{d} t}\left\|\nabla E_{c}^{h}\right\|^{2}+\frac{1}{2}\left\|\nabla E_{w}^{h}\right\|^{2}+\frac{1}{2} \frac{\mathrm{d}}{\mathrm{d} t}\left\|E_{\mathbf{u}}^{h}\right\|^{2}+\frac{1}{2}\left\|\nabla E_{\mathbf{u}}^{h}\right\|^{2} \leqslant \widehat{C}\left(h^{2}+\left\|\nabla E_{c}^{h}\right\|^{2}+\left\|E_{\mathbf{u}}^{h}\right\|^{2}\right) .
$$


LEMMA 3.6 For all $t \in[0, T]$ we have

$$
\left\|E_{c}^{h}(t)\right\|_{1}^{2}+\left\|E_{\mathbf{u}}^{h}(t)\right\|^{2}+\int_{0}^{T}\left(\left\|\nabla E_{w}^{h}\right\|^{2}+\left\|\nabla E_{\mathbf{u}}^{h}\right\|^{2}\right) \mathrm{d} t \leqslant C\left(h^{2}+\left\|\nabla E_{c}^{h}(0)\right\|^{2}+\left\|E_{\mathbf{u}}^{h}(0)\right\|^{2}\right) .
$$

Proof. Applying Gronwall's inequality to 3.33 and noting 2.14, 2.22) and (3.3b) we obtain the required result.

THEOREM 3.2 If

$$
\left\|c(0)-c_{h}(0)\right\|_{1}+\left\|\mathbf{u}(0)-\mathbf{u}_{h}(0)\right\| \leqslant C h
$$

then

$$
\left\|c-c_{h}\right\|_{L^{\infty}\left(H^{1}\right)}+\left\|\mathbf{u}-\mathbf{u}_{h}\right\|_{L^{\infty}\left(\mathbf{L}^{2}\right)}+\left\|\nabla\left(w-w_{h}\right)\right\|_{L^{2}\left(L^{2}\right)}+\left\|\mathbf{u}-\mathbf{u}_{h}\right\|_{L^{2}\left(\mathbf{H}^{1}\right)} \leqslant C h .
$$

Proof. The desired result is a direct consequence of Lemma 3.6, 2.26 and 2.28).

REMARK 3.3 We note that in the above error bound $\gamma$ enters in the form $\exp \left(\gamma^{-2}\right)$. Since $\gamma$ is required to be a small parameter, ideally one would like to obtain an estimate that depends polynomially on $\gamma^{-1}$. Such an estimate has been proved in [20] in which a spectrum estimate result for the linearized Cahn-Hilliard operator is used; however, as is mentioned in [19], we know of no such spectrum estimate for the linearized operator associated with the coupled system $(1.1 \mathrm{a}-\mathrm{d})$.

\section{Fully discrete scheme}

In this section we prove the convergence of a fully discrete finite element approximation $\left(\mathbf{P}_{h, \tau}\right)$, of $(\mathbf{P})$.

We define $\mathcal{T}^{h}$ to be as in Section 2.2 and in addition we let $0=t_{0}<t_{1}<\cdots<t_{N-1}<t_{N}=T$ be a partitioning of $[0, T]$ into possibly variable time steps $\tau_{n}:=t_{n}-t_{n-1}$, for $n=1 \rightarrow N$. We set $\tau:=\max _{n=1 \rightarrow N} \tau_{n}$.

Finally, for simplicity of notation we set

$$
\delta_{\tau} c_{h}^{n}:=\frac{c_{h}^{n}-c_{h}^{n-1}}{\tau_{n}} \quad \text { and } \quad \delta_{\tau} \mathbf{u}_{h}^{n}:=\frac{\mathbf{u}_{h}^{n}-\mathbf{u}_{h}^{n-1}}{\tau_{n}} .
$$

We now define a fully discrete finite element approximation of $(\mathbf{P})$ :

$\left(\mathbf{P}_{h, \tau}\right)$ For $n \geqslant 1$ find $\left\{c_{h}^{n}, w_{h}^{n}, \mathbf{u}_{h}^{n}\right\} \in V^{h} \times S^{h} \times \mathbf{W}_{0}^{h}$ such that

$$
\begin{array}{cc}
\left(\delta_{\tau} c_{h}^{n}, \chi\right)_{h}+\left(\nabla w_{h}^{n}, \nabla \chi\right)=\mathbf{a}\left(c_{h}^{n-1}, \chi, \mathbf{u}_{h}^{n-1}\right) & \forall \chi \in S^{h}, \\
\left(w_{h}^{n}, \chi\right)_{h}=\gamma^{2}\left(\nabla c_{h}^{n}, \nabla \chi\right)+\left(\phi\left(c_{h}^{n}\right)-c_{h}^{n-1}, \chi\right)_{h} & \forall \chi \in S^{h}, \\
\left(\delta_{\tau} \mathbf{u}_{h}^{n}, \chi\right)+\left(\nabla \mathbf{u}_{h}^{n}: \nabla \chi\right)+B\left(\mathbf{u}_{h}^{n-1}, \mathbf{u}_{h}^{n}, \chi\right)+\mathbf{a}\left(c_{h}^{n-1}, w_{h}^{n}, \chi\right)=0 & \forall \chi \in \mathbf{W}_{0}^{h}, \\
c_{h}^{0}(x)=Q^{h}\left(c_{0}(x)\right) \in V^{h} \cap L^{\infty}(\Omega), \quad \mathbf{u}_{h}^{0}(x)=\mathbf{P}^{h}\left(\mathbf{u}_{0}(x)\right) \in \mathbf{W}_{0}^{h} & \forall x \in \Omega,
\end{array}
$$

where $\phi(s)=s^{3}$. 
REMARK 4.1 From (4.1d), 2.24, 2.28) and 2.14) we have

$$
\left(\Phi\left(c_{h}^{0}(\cdot)\right), 1\right)_{h}+\left\|c_{h}^{0}(\cdot)\right\|_{1}+\left\|\mathbf{u}_{h}^{0}(\cdot)\right\| \leqslant C .
$$

LEMMA 4.1 For all $h, \tau_{n}>0$ there exists a unique solution $\left\{\mathbf{u}_{h}^{n}, c_{h}^{n}, w_{h}^{n}\right\}$ to the $n$-th step of $\left(\mathbf{P}_{h, \tau}\right)$.

Proof. In order to prove existence of a unique solution $\left\{c_{h}^{n}, w_{h}^{n}\right\} \in V^{h} \times S^{h}$ to $4.1 \mathrm{a}$ b) first we set $\chi=\widehat{\mathcal{G}}^{h} \chi$ in $4.1 \mathrm{a}$ and note 2.2 to obtain

$$
\begin{aligned}
\left(\delta_{\tau} c_{h}^{n}, \widehat{\mathcal{G}}^{h} \chi\right)_{h}+\left(\nabla w_{h}^{n}, \nabla \widehat{\mathcal{G}}^{h} \chi\right) & =\left(c_{h}^{n-1}, \nabla\left(\widehat{\mathcal{G}}^{h} \chi\right) \cdot \mathbf{u}_{h}^{n-1}\right) \\
& =-\left(\nabla \cdot\left(c_{h}^{n-1} \mathbf{u}_{h}^{n-1}\right), \widehat{\mathcal{G}}^{h} \chi\right) \quad \forall \chi \in V^{h} .
\end{aligned}
$$

Using (4.3) and 2.30a,b) we obtain

$$
\left(w_{h}^{n}, \chi\right)_{h}=\left(\nabla w_{h}^{n}, \nabla \widehat{\mathcal{G}}^{h} \chi\right)=-\left(\widehat{\mathcal{G}}^{h}\left[\delta_{\tau} c_{h}^{n}\right], \chi\right)_{h}-\left(\mathcal{G}^{h}\left[\nabla \cdot\left(c_{h}^{n-1} \mathbf{u}_{h}^{n-1}\right)\right], \chi\right)_{h} \quad \forall \chi \in V^{h} .
$$

Hence $4.1 \mathrm{a}, \mathrm{b})$ can be restated as: Find $c_{h}^{n} \in V^{h}$ such that for all $\chi \in V^{h}$

$$
\gamma^{2}\left(\nabla c_{h}^{n}, \nabla \chi\right)+\left(\phi\left(c_{h}^{n}\right)+\frac{1}{\tau_{n}} \widehat{\mathcal{G}}^{h}\left[\delta_{\tau} c_{h}^{n}\right], \chi\right)_{h}=\left(c_{h}^{n-1}-\mathcal{G}^{h}\left[\nabla \cdot\left(c_{h}^{n-1} \mathbf{u}_{h}^{n-1}\right)\right], \chi\right)_{h} .
$$

There exists $c_{h}^{n} \in V^{h}$ solving 4.5 since, on noting 2.30b), this is the Euler-Lagrange equation of the convex minimization problem

$$
\min _{z^{h} \in V^{h}}\left\{\frac{\gamma^{2}}{2}\left\|\nabla z^{h}\right\|^{2}+\frac{1}{4}\left(\left(z^{h}\right)^{4}, 1\right)_{h}+\frac{1}{2 \tau_{n}}\left\|\nabla \widehat{\mathcal{G}}^{h}\left(z^{h}-c_{h}^{n-1}\right)\right\|^{2}-\left(c_{h}^{n-1}-\mathcal{G}^{h}\left[\nabla \cdot\left(c_{h}^{n-1} \mathbf{u}_{h}^{n-1}\right)\right], z^{h}\right)_{h}\right\} .
$$

Therefore on noting 4.4, we have existence of a unique solution $\left\{c_{h}^{n}, w_{h}^{n}\right\} \in V^{h} \times S^{h}$ to 4.1a. b). The existence of a unique solution $\mathbf{u}_{h}^{n} \in \mathbf{W}_{0}^{h}$ to $4.1 \mathrm{c}$ follows from standard results.

LEMMA 4.2 Let $c_{h}^{n-1} \in V^{h}$. Then for $\tau_{n} \leqslant C h /\left(4\left\|c_{h}^{n-1}\right\|_{0,4}^{2}\right)$ we have

$$
\begin{aligned}
\mathcal{E}\left(c_{h}^{n}, \mathbf{u}_{h}^{n}\right)+\frac{1}{2} \gamma^{2}\left\|\nabla\left(c_{h}^{n}-c_{h}^{n-1}\right)\right\|^{2}+\frac{1}{4}\left\|\mathbf{u}_{h}^{n}-\mathbf{u}_{h}^{n-1}\right\|^{2}+\frac{1}{2} \tau_{n}\left\|\nabla w_{h}^{n}\right\|^{2} & +\tau_{n}\left\|\nabla \mathbf{u}_{h}^{n}\right\|^{2} \\
& \leqslant \mathcal{E}\left(c_{h}^{n-1}, \mathbf{u}_{h}^{n-1}\right)
\end{aligned}
$$

where

$$
\mathcal{E}\left(c_{h}^{n}, \mathbf{u}_{h}^{n}\right):=\frac{1}{2} \gamma^{2}\left\|\nabla c_{h}^{n}\right\|^{2}+\left(\Phi\left(c_{h}^{n}\right), 1\right)_{h}+\frac{1}{2}\left\|\mathbf{u}_{h}^{n}\right\|^{2} .
$$

Proof. Choosing $\chi \equiv w_{h}^{n}$ in $4.1 \mathrm{a}$ and $\chi \equiv c_{h}^{n}-c_{h}^{n-1}$ in $4.1 \mathrm{~b}$ yields respectively

$$
\left(c_{h}^{n}-c_{h}^{n-1}, w_{h}^{n}\right)_{h}+\tau_{n}\left(\nabla w_{h}^{n}, \nabla w_{h}^{n}\right)=\tau_{n} \mathbf{a}\left(c_{h}^{n-1}, w_{h}^{n}, \mathbf{u}_{h}^{n-1}\right)
$$

and

$$
\gamma^{2}\left(\nabla c_{h}^{n}, \nabla\left[c_{h}^{n}-c_{h}^{n-1}\right]\right)=\left(w_{h}^{n}-\phi\left(c_{h}^{n}\right)+c_{h}^{n-1}, c_{h}^{n}-c_{h}^{n-1}\right)_{h} .
$$

Combining (4.8) and 4.9, using the elementary identity

$$
2 r(r-s)=\left(r^{2}-s^{2}\right)+(r-s)^{2} \quad \forall r, s \in \mathbb{R},
$$


and noting the following result from [18]:

$$
(\phi(r)-s)(r-s) \geqslant \Phi(r)-\Phi(s)
$$

we obtain

$$
\begin{aligned}
\frac{1}{2} \gamma^{2}\left\|\nabla\left(c_{h}^{n}-c_{h}^{n-1}\right)\right\|^{2}+ & \frac{1}{2} \gamma^{2}\left\|\nabla c_{h}^{n}\right\|^{2}+\left(\Phi\left(c_{h}^{n}\right), 1\right)_{h}+\tau_{n}\left\|\nabla w_{h}^{n}\right\|^{2} \\
& \leqslant\left(\Phi\left(c_{h}^{n-1}\right), 1\right)_{h}+\frac{1}{2} \gamma^{2}\left\|\nabla c_{h}^{n-1}\right\|^{2}+\tau_{n} \mathbf{a}\left(c_{h}^{n-1}, w_{h}^{n}, \mathbf{u}_{h}^{n-1}\right) .
\end{aligned}
$$

Choosing $\chi \equiv \mathbf{u}_{h}^{n}$ in (4.1c) and noting (4.10) and 2.4 we obtain

$$
\frac{1}{2}\left\|\mathbf{u}_{h}^{n}-\mathbf{u}_{h}^{n-1}\right\|^{2}+\frac{1}{2}\left\|\mathbf{u}_{h}^{n}\right\|^{2}-\frac{1}{2}\left\|\mathbf{u}_{h}^{n-1}\right\|^{2}+\tau_{n}\left\|\nabla \mathbf{u}_{h}^{n}\right\|^{2}+\tau_{n} \mathbf{a}\left(c_{h}^{n-1}, w_{h}^{n}, \mathbf{u}_{h}^{n}\right)=0 .
$$

Combining (4.12), (4.13) and noting (4.7) we have

$$
\begin{aligned}
\mathcal{E}\left(c_{h}^{n}, \mathbf{u}_{h}^{n}\right)+\frac{1}{2} \gamma^{2}\left\|\nabla\left(c_{h}^{n}-c_{h}^{n-1}\right)\right\|^{2}+ & \frac{1}{2}\left\|\mathbf{u}_{h}^{n}-\mathbf{u}_{h}^{n-1}\right\|^{2}+\tau_{n}\left\|\nabla w_{h}^{n}\right\|^{2}+\tau_{n}\left\|\nabla \mathbf{u}_{h}^{n}\right\|^{2} \\
& \leqslant \mathcal{E}\left(c_{h}^{n-1}, \mathbf{u}_{h}^{n-1}\right)+\tau_{n} \mathbf{a}\left(c_{h}^{n-1}, w_{h}^{n}, \mathbf{u}_{h}^{n-1}-\mathbf{u}_{h}^{n}\right) .
\end{aligned}
$$

Noting 2.32a and choosing $\tau_{n}$ such that $\tau_{n} \leqslant C h /\left(4\left\|c_{h}^{n-1}\right\|_{0,4}^{2}\right)$ we have

$$
\begin{aligned}
\tau_{n}\left|\mathbf{a}\left(c_{h}^{n-1}, w_{h}^{n}, \mathbf{u}_{h}^{n-1}-\mathbf{u}_{h}^{n}\right)\right| & \leqslant \tau_{n}\left\|c_{h}^{n-1}\right\|_{0,4}\left\|\nabla w_{h}^{n}\right\|\left\|\mathbf{u}_{h}^{n}-\mathbf{u}_{h}^{n-1}\right\|_{0,4} \\
& \leqslant \frac{1}{2} \tau_{n}\left\|c_{h}^{n-1}\right\|_{0,4}^{2}\left\|\mathbf{u}_{h}^{n}-\mathbf{u}_{h}^{n-1}\right\|_{0,4}^{2}+\frac{1}{2} \tau_{n}\left\|\nabla w_{h}^{n}\right\|^{2} \\
& \leqslant C \tau_{n} h^{-1}\left\|c_{h}^{n-1}\right\|_{0,4}^{2}\left\|\mathbf{u}_{h}^{n}-\mathbf{u}_{h}^{n-1}\right\|^{2}+\frac{1}{2} \tau_{n}\left\|\nabla w_{h}^{n}\right\|^{2} \\
& \leqslant \frac{1}{4}\left\|\mathbf{u}_{h}^{n}-\mathbf{u}_{h}^{n-1}\right\|^{2}+\frac{1}{2} \tau_{n}\left\|\nabla w_{h}^{n}\right\|^{2},
\end{aligned}
$$

and hence from (4.14) and 4.15) we conclude 4.6.

THEOREM 4.1 For all $h>0$ and for all time partitions $\left\{\tau_{n}\right\}_{n=1}^{N}$ such that $\tau_{n} \leqslant C h /\left(4\left\|c_{h}^{n-1}\right\|_{0,4}^{2}\right)$, the solution $\left\{c_{h}^{n}, w_{h}^{n}, \mathbf{u}_{h}^{n}\right\}_{n=1}^{N}$ to $\left(\mathbf{P}_{h, \tau}\right)$ is such that

$$
\begin{array}{r}
\max _{n=1 \rightarrow N}\left[\gamma^{2}\left\|c_{h}^{n}\right\|_{1}^{2}+\left\|\mathbf{u}_{h}^{n}\right\|^{2}+\left(\Phi\left(c_{h}^{n}\right), 1\right)_{h}\right]+\sum_{n=1}^{N}\left[\gamma^{2}\left\|\nabla\left(c_{h}^{n}-c_{h}^{n-1}\right)\right\|^{2}+\left\|\mathbf{u}_{h}^{n}-\mathbf{u}_{h}^{n-1}\right\|^{2}\right] \\
+\sum_{n=1}^{N} \tau_{n}\left[\left\|\nabla w_{h}^{n}\right\|^{2}+\left\|\nabla \mathbf{u}_{h}^{n}\right\|^{2}\right] \leqslant C\left(\left\|\nabla c_{h}^{0}\right\|^{2}+\left\|\mathbf{u}_{h}^{0}\right\|^{2}+\left(\Phi\left(c_{h}^{0}\right), 1\right)_{h}\right) \leqslant C .
\end{array}
$$

In addition

$$
\sum_{n=1}^{N} \tau_{n}\left\|\nabla \mathcal{G}\left[\delta_{\tau} c_{h}^{n}\right]\right\|^{2} \leqslant C .
$$

Proof. The result (4.16a) follows by summing (4.6) from $n=1 \rightarrow N$ and noting (2.22) and 4.2). From 2.5, 2.23, (4.1a), 2.8) and 2.24) we have for any $\eta \in H^{1}(\Omega)$,

$$
\begin{aligned}
\left(\nabla \mathcal{G}\left[\delta_{\tau} c_{h}^{n}\right], \nabla \eta\right) & =\left(\delta_{\tau} c_{h}^{n}, \eta\right)=\left(\delta_{\tau} c_{h}^{n}, Q^{h} \eta\right)_{h}=-\left(\nabla w_{h}^{n}, \nabla\left[Q^{h} \eta\right]\right)+\mathbf{a}\left(c_{h}^{n-1}, Q^{h} \eta, \mathbf{u}_{h}^{n-1}\right) \\
& \leqslant\left[\left\|\nabla w_{h}^{n}\right\|+\left\|\mathbf{u}_{h}^{n-1}\right\|_{1}\left\|c_{h}^{n-1}\right\|_{1}\right]\left\|\nabla Q^{h} \eta\right\| \\
& \leqslant C\left[\left\|\nabla w_{h}^{n}\right\|+\left\|\mathbf{u}_{h}^{n-1}\right\|_{1}\left\|c_{h}^{n-1}\right\|_{1}\right]\|\nabla \eta\| .
\end{aligned}
$$

The bound in 4.16b then follows from 4.17) and 4.16a. 
REMARK 4.2 We note that the bound $\tau_{n} \leqslant C h /\left(4\left\|c_{h}^{n-1}\right\|_{0,4}^{2}\right)$ in Lemma 4.2 and Theorem 4.1 is attainable since from 4.16a and 2.9 we have $\left\|c_{h}^{n-1}\right\|_{0,4} \leqslant C\left\|c_{h}^{n-1}\right\|_{1} \leqslant C$.

We now consider the convergence of $\left(\mathbf{P}_{h, \tau}\right)$ as $h$ and $\tau$ tend to zero. To this end we let

$$
\begin{array}{ll}
c_{h, \tau}(t):=\frac{t-t_{n-1}}{\tau_{n}} c_{h}^{n}+\frac{t_{n}-t}{\tau_{n}} c_{h}^{n-1}, \quad t \in\left[t_{n-1}, t_{n}\right], n \geqslant 1, \\
c_{h, \tau}^{+}(t):=c_{h}^{n}, \quad c_{h, \tau}^{-}(t):=c_{h}^{n-1}, \quad t \in\left(t_{n-1}, t_{n}\right], n \geqslant 1 .
\end{array}
$$

We note for future reference that

$$
c_{h, \tau}-c_{h, \tau}^{ \pm}=\left(t-t_{n}^{ \pm}\right) \partial_{t} c_{h, \tau}, \quad t \in\left(t_{n-1}, t_{n}\right), n \geqslant 1,
$$

where $t_{n}^{+}:=t_{n}$ and $t_{n}^{-}:=t_{n-1}$. We also introduce

$$
\bar{\tau}(t):=\tau_{n}, \quad t \in\left(t_{n-1}, t_{n}\right], n \geqslant 1
$$

Using the above notation, and introducing analogous notation for $w_{h, \tau}$ and $\mathbf{u}_{h, \tau}$, we can restate $\left(\mathbf{P}_{h, \tau}\right)$ as:

Find $\left\{c_{h, \tau}, w_{h, \tau}, \mathbf{u}_{h, \tau}\right\} \in L^{2}\left(0, T ; V^{h}\right) \times L^{2}\left(0, T ; S^{h}\right) \times L^{2}\left(0, T ; \mathbf{W}_{0}^{h}\right)$ such that

$$
\begin{gathered}
\int_{0}^{T}\left(\left(\partial_{t} c_{h, \tau}, \chi\right)_{h}+\left(\nabla w_{h, \tau}^{+}, \nabla \chi\right)-\mathbf{a}\left(c_{h, \tau}^{-}, \chi, \mathbf{u}_{h, \tau}^{-}\right)\right) \mathrm{d} t=0 \quad \forall \chi \in L^{2}\left(0, T ; S^{h}\right) \\
\int_{0}^{T}\left(w_{h, \tau}^{+}, \chi\right)_{h} \mathrm{~d} t=\int_{0}^{T}\left(\gamma^{2}\left(\nabla c_{h, \tau}^{+}, \nabla \chi\right)+\left(\phi\left(c_{h, \tau}^{+}\right)-c_{h, \tau}^{-}, \chi\right)_{h}\right) \mathrm{d} t \quad \forall \chi \in L^{2}\left(0, T ; S^{h}\right) \\
\int_{0}^{T}\left(\left(\partial_{t} \mathbf{u}_{h}, \chi\right)+\left(\nabla \mathbf{u}_{h, \tau}^{+}: \nabla \chi\right)+B\left(\mathbf{u}_{h, \tau}^{-}, \mathbf{u}_{h, \tau}^{+}, \chi\right)+\mathbf{a}\left(c_{h, \tau}^{-}, w_{h, \tau}^{+}, \chi\right)\right) \mathrm{d} t=0 \\
\forall \chi \in L^{2}\left(0, T ; \mathbf{W}_{0}^{h}\right) .
\end{gathered}
$$

LEMmA 4.3 There exists a subsequence of $\left\{c_{h, \tau}, w_{h, \tau}, \mathbf{u}_{h, \tau}\right\}_{h}$, where $\left\{c_{h, \tau}, w_{h, \tau}, \mathbf{u}_{h, \tau}\right\}$ solve $\left(\mathbf{P}_{h, \tau}\right)$, and functions

$$
\left\{\begin{array}{l}
c \in L^{\infty}(0, T ; V) \cap H^{1}\left(0, T ;\left(H^{1}(\Omega)\right)^{\prime}\right), \quad w \in L^{2}\left(0, T ; H^{1}(\Omega)\right) \\
\mathbf{u} \in L^{\infty}\left(0, T ; L^{2}(\Omega)\right) \cap L^{2}\left(0, T ; \mathbf{W}_{0}\right)
\end{array}\right.
$$

with $c(\cdot, 0)=c_{0}(\cdot)$ in $L^{2}(\Omega)$ and $\mathbf{u}(\cdot, 0)=\mathbf{u}_{0}(\cdot)$ in $\mathbf{L}^{2}(\Omega)$ are such that as $h \rightarrow 0$,

$$
\begin{array}{ll}
c_{h, \tau}, c_{h, \tau}^{ \pm} \rightarrow c & \text { weak-* in } L^{\infty}\left(0, T ; H^{1}(\Omega)\right), \\
c_{h, \tau}, c_{h, \tau}^{ \pm} \rightarrow c & \text { strongly in } L^{2}\left(0, T ; L^{2}(\Omega)\right), \\
\mathcal{G}\left(\partial_{t} c_{h, \tau}\right) \rightarrow \mathcal{G}\left(\partial_{t} c\right) & \text { weakly in } L^{2}\left(0, T ; H^{1}(\Omega)\right), \\
w_{h, \tau}, w_{h, \tau}^{ \pm} \rightarrow w & \text { weakly in } L^{2}\left(0, T ; H^{1}(\Omega)\right), \\
\mathbf{u}_{h, \tau}, \mathbf{u}_{h, \tau}^{ \pm} \rightarrow \mathbf{u} & \text { weakly in } L^{2}\left(0, T ; \mathbf{H}^{1}(\Omega)\right) .
\end{array}
$$


Proof. Noting the definitions 4.18a,b), 4.20, the bounds in Theorem 4.1. 4.16b together with (2.6) and (4.2) imply that

$$
\begin{aligned}
\left\|c_{h, \tau}^{( \pm)}\right\|_{L^{\infty}\left(H^{1}\right)}^{2}+\left\|\mathbf{u}_{h, \tau}^{( \pm)}\right\|_{L^{\infty}\left(\mathbf{L}^{2}\right)}^{2}+\left\|\bar{\tau}^{1 / 2} \partial_{t} c_{h, \tau}\right\|_{L^{2}\left(H^{1}\right)}^{2}+\left\|\bar{\tau}^{1 / 2} \partial_{t} \mathbf{u}_{h}\right\|_{L^{2}\left(\mathbf{L}^{2}\right)}^{2} \\
+\left\|\nabla w_{h, \tau}^{+}\right\|_{L^{2}\left(L^{2}\right)}^{2}+\left\|\mathbf{u}_{h, \tau}^{+}\right\|_{L^{2}\left(\mathbf{H}^{1}\right)}^{2}+\left\|\mathcal{G}\left(\partial_{t} c_{h, \tau}\right)\right\|_{L^{2}\left(H^{1}\right)}^{2} \leqslant C .
\end{aligned}
$$

Furthermore, we deduce from (4.19) and (4.24) that

$$
\left\|c_{h, \tau}-c_{h, \tau}^{ \pm}\right\|_{L^{2}\left(H^{1}\right)}^{2} \leqslant\left\|\bar{\tau} \partial_{t} c_{h, \tau}\right\|_{L^{2}\left(H^{1}\right)}^{2} \leqslant C \tau
$$

and

$$
\left\|\mathbf{u}_{h, \tau}-\mathbf{u}_{h, \tau}^{ \pm}\right\|_{L^{2}\left(\mathbf{L}^{2}\right)}^{2} \leqslant\left\|\bar{\tau} \partial_{t} \mathbf{u}_{h, \tau}\right\|_{L^{2}\left(\mathbf{L}^{2}\right)}^{2} \leqslant C \tau .
$$

Hence on noting 4.24, 4.25a,b) and 2.7a we can choose a subsequence $\left\{c_{h, \tau}, w_{h, \tau}, \mathbf{u}_{h, \tau}\right\}_{h}$ such that the convergence results (4.22) and (4.23a-f) hold. Then (4.22) yields on noting (2.7b), 2.24) and $(2.28)$ that the subsequences satisfy the additional initial conditions.

We follow the arguments used in [28] to prove the following lemma.

LEMMA 4.4 We have

$$
\mathbf{u}_{h, \tau}, \mathbf{u}_{h, \tau}^{ \pm} \rightarrow \mathbf{u} \quad \text { strongly in } L^{2}\left(0, T ; \mathbf{L}^{2}(\Omega)\right) .
$$

Proof. Since $\left\{\mathbf{u}_{h, \tau}\right\}_{h}$ is bounded in $L^{\infty}\left(0, T ; \mathbf{L}^{2}(\Omega)\right) \cap L^{2}\left(0, T ; \mathbf{H}^{1}(\Omega)\right)$, a result of J.-L. Lions [28, 29] states that compactness of the sequence $\left\{\mathbf{u}_{h, \tau}^{ \pm}\right\}_{h}$ in $L^{2}\left(0, T ; \mathbf{L}^{2}(\Omega)\right)$ will follow if

$$
\int_{\delta}^{T}\left\|\mathbf{u}_{h, \tau}^{+}(t)-\mathbf{u}_{h, \tau}^{+}(t-\delta)\right\|_{\mathbf{L}^{2}(\Omega)}^{2} \leqslant C \delta^{\alpha}
$$

for $0 \leqslant \delta \leqslant T$ and some $\alpha>0$. Since $\left\{\mathbf{u}_{h, \tau}^{+}\right\}_{h}$ are piecewise constant in time it suffices to take $\delta$ to be a multiple of the time step $\tau_{n}$. Writing $(t-\delta, t)=\left(t^{m}, t^{n}\right)$ and setting $\mathbf{v}(t):=\mathbf{u}_{h, \tau}^{+}\left(t^{n}\right)-\mathbf{u}_{h, \tau}^{+}\left(t^{m}\right)=$ $\mathbf{v}^{m n}$ in $4.1 \mathrm{c}$ yields

$$
\begin{aligned}
\left(\mathbf{u}_{h, \tau}^{+}\left(t^{n}\right)-\mathbf{u}_{h, \tau}^{+}\left(t^{m}\right), \mathbf{v}^{m n}\right)= & -\int_{t^{m}}^{t^{n}}\left[\left(\nabla \mathbf{u}_{h, \tau}^{+}: \nabla \mathbf{v}^{m n}\right)+B\left(\mathbf{u}_{h, \tau}^{+}\left(\cdot-\tau_{n}\right), \mathbf{u}_{h, \tau}^{+}, \mathbf{v}^{m n}\right)\right] \mathrm{d} s \\
& -\int_{t^{m}}^{t^{n}} \mathbf{a}\left(c_{h, \tau}\left(\cdot-\tau_{n}\right), w_{h, \tau}^{+}, \mathbf{v}^{m n}\right) \mathrm{d} s .
\end{aligned}
$$

Using 2.8, 4.24, Hölder's inequality and 4.16a we have

$$
\begin{aligned}
& \mid \int_{t^{m}}^{t^{n}}\left(\left(\nabla \mathbf{u}_{h, \tau}^{+}: \nabla \mathbf{v}^{m n}\right)\right.\left.-\mathbf{a}\left(c_{h, \tau}\left(\cdot-\tau_{n}\right), w_{h, \tau}^{+}, \mathbf{v}^{m n}\right)\right) \mathrm{d} s \mid \\
& \leqslant \int_{t^{m}}^{t^{n}}\left(\left\|\nabla \mathbf{u}_{h, \tau}^{+}\right\|\left\|\nabla \mathbf{v}^{m n}\right\|+\left\|c_{h, \tau}\left(\cdot-\tau_{n}\right)\right\|\left\|_{1}\right\| \nabla w_{h, \tau}^{+}\|\| \mathbf{v}^{m n} \|_{1}\right) \mathrm{d} s \\
& \leqslant C\left\|\mathbf{v}^{m n}\right\|_{1} \int_{t^{m}}^{t^{n}}\left(\left\|\nabla \mathbf{u}_{h, \tau}^{+}\right\|+\left\|\nabla w_{h, \tau}^{+}\right\|\right) \\
& \leqslant C\left\|\mathbf{v}^{m n}\right\|_{1}\left(t^{n}-t^{m}\right)^{1 / 2}\left(\left\|\nabla \mathbf{u}_{h, \tau}^{+}\right\|_{L^{2}\left(\mathbf{L}^{2}\right)}+\left\|\nabla w_{h, \tau}^{+}\right\|_{L\left(\mathbf{L}^{2}\right)}\right)
\end{aligned}
$$


and similarly noting 2.9 ) and 4.24 we obtain

$$
\begin{array}{rl}
\mid \int_{t^{m}}^{t^{n}} & B\left(\mathbf{u}_{h, \tau}^{+}\left(\cdot-\tau_{n}\right), \mathbf{u}_{h, \tau}^{+}, \mathbf{v}^{m n}\right) \mathrm{d} s \mid \\
\leqslant & \int_{t^{m}}^{t^{n}}\left\|\mathbf{u}_{h, \tau}^{+}\left(\cdot-\tau_{n}\right)\right\|_{0,4}\left(\left\|\nabla \mathbf{u}_{h, \tau}^{+}\right\|\left\|\mathbf{v}^{m n}\right\|_{0,4}+\left\|\nabla \mathbf{v}^{m n}\right\|\left\|\mathbf{u}_{h, \tau}^{+}\right\|_{0,4}\right) \mathrm{d} s \\
\leqslant & C \int_{t^{m}}^{t^{n}}\left\|\mathbf{u}_{h, \tau}^{+}\left(\cdot-\tau_{n}\right)\right\|^{1 / 2}\left\|\mathbf{u}_{h, \tau}^{+}\left(\cdot-\tau_{n}\right)\right\|_{1}^{1 / 2} \\
& \times\left(\left\|\nabla \mathbf{u}_{h, \tau}^{+}\right\|\left\|\mathbf{v}^{m n}\right\|^{1 / 2}\left\|\mathbf{v}^{m n}\right\|_{1}^{1 / 2}+\left\|\nabla \mathbf{v}^{m n}\right\|\left\|\mathbf{u}_{h, \tau}^{+}\right\|^{1 / 2}\left\|\mathbf{u}_{h, \tau}^{+}\right\|_{1}^{1 / 2}\right) \mathrm{d} s \\
\leqslant & C \int_{t^{m}}^{t^{n}}\left\|\mathbf{u}_{h, \tau}^{+}\left(\cdot-\tau_{n}\right)\right\|_{1}^{1 / 2}\left(\left\|\nabla \mathbf{u}_{h, \tau}^{+}\right\|\left\|\mathbf{v}^{m n}\right\|_{1}^{1 / 2}+\left\|\nabla \mathbf{v}^{m n}\right\|\left\|\mathbf{u}_{h, \tau}^{+}\right\|_{1}^{1 / 2}\right) \mathrm{d} s \\
\leqslant & C\left(t^{n}-t^{m}\right)^{1 / 4}\left\|\mathbf{v}^{m n}\right\|_{1}^{1 / 2}\left\|\mathbf{u}_{h, \tau}^{+}\right\|_{L^{2}\left(\mathbf{H}^{1}\right)}^{3 / 2}+C\left(t^{n}-t^{m}\right)^{1 / 2}\left\|\nabla \mathbf{v}^{m n}\right\|\left\|\mathbf{u}_{h, \tau}^{+}\right\|_{L^{2}\left(\mathbf{H}^{1}\right)} .
\end{array}
$$

Integrating (4.28) with respect to $t^{n} \in(\delta, T)$, using (4.29) and 4.30) we obtain 4.27), and noting (4.25b) gives the required result.

LEMMA 4.5 Let $\mathbf{v}_{h}, \eta_{h}$ and $\xi_{h}$ be such that

$$
\left\|\xi_{h} \nabla \eta_{h}\right\|_{L^{2}\left(\mathbf{L}^{2}\right)} \leqslant C, \quad\left\|\mathbf{v}_{h} \xi_{h}\right\|_{L^{2}\left(\mathbf{L}^{2}\right)} \leqslant C, \quad\left\|\mathbf{v}_{h} \cdot \nabla \eta_{h}\right\|_{L^{2}\left(L^{2}\right)} \leqslant C
$$

and

$$
\begin{array}{ll}
\mathbf{v}_{h} \rightarrow \mathbf{v} & \text { strongly in } L^{2}\left(0, T ; \mathbf{L}^{2}(\Omega)\right), \\
\xi_{h} \rightarrow \xi & \text { strongly in } L^{2}\left(0, T ; L^{2}(\Omega)\right), \\
\eta_{h} \rightarrow \eta & \text { weakly in } L^{2}\left(0, T ; H^{1}(\Omega)\right) .
\end{array}
$$

Then

$$
\int_{0}^{T} \mathbf{a}\left(\xi_{h}, \eta_{h}, \mathbf{v}_{h}\right) \mathrm{d} t \rightarrow \int_{0}^{T} \mathbf{a}(\xi, \eta, \mathbf{v}) \mathrm{d} t
$$

Proof. We have

$$
\begin{aligned}
& \left|\int_{0}^{T} \mathbf{a}\left(\xi_{h}, \eta_{h}, \mathbf{v}_{h}\right)-\mathbf{a}(\xi, \eta, \mathbf{v}) \mathrm{d} t\right| \\
& \quad \leqslant\left|\int_{0}^{T} \mathbf{a}\left(\xi_{h}, \eta_{h}, \mathbf{v}_{h}-\mathbf{v}\right) \mathrm{d} t\right|+\left|\int_{0}^{T} \mathbf{a}\left(\xi_{h}-\xi, \eta_{h}, \mathbf{v}\right) \mathrm{d} t\right|+\left|\int_{0}^{T} \mathbf{a}\left(\xi, \eta_{h}-\eta, \mathbf{v}\right) \mathrm{d} t\right| \\
& \quad \leqslant\left\|\mathbf{v}_{h}-\mathbf{v}\right\|_{L^{2}\left(\mathbf{L}^{2}\right)}\left\|\xi_{h} \nabla \eta_{h}\right\|_{L^{2}\left(\mathbf{L}^{2}\right)}+\left\|\mathbf{v} \cdot \nabla \eta_{h}\right\|_{L^{2}\left(L^{2}\right)}\left\|\xi_{h}-\xi\right\|_{L^{2}\left(L^{2}\right)}+\left|\int_{0}^{T} \mathbf{a}\left(\xi, \eta_{h}-\eta, \mathbf{v}\right) \mathrm{d} t\right|
\end{aligned}
$$

and the desired result follows from 4.31) and 4.32).

THEOREM 4.2 There exists a subsequence of $\left\{c_{h, \tau}, w_{h, \tau}, \mathbf{u}_{h, \tau}\right\}_{h}$, where $\left\{c_{h, \tau}, w_{h, \tau}, \mathbf{u}_{h, \tau}\right\}$ solve $\left(\mathbf{P}_{h, \tau}\right)$, and functions $\{c, w, \mathbf{u}\}$ satisfying 4.22 . In addition, as $\left.h \rightarrow 0,4.23 \mathrm{a}-\mathrm{e}\right)$ hold. Furthermore, $\{c, w, \mathbf{u}\}$ fulfil $c(\cdot, 0)=c_{0}(\cdot)$ in $L^{2}(\Omega), \mathbf{u}(\cdot, 0)=\mathbf{u}_{0}(\cdot)$ in $\mathbf{L}^{2}(\Omega)$ and satisfy for all $\eta \in$ 
$L^{2}\left(0, T ; H^{1}(\Omega)\right)$ and all $\mathbf{v} \in L^{2}\left(0, T ; \mathbf{W}_{0}\right) \cap H^{1}\left(0, T ; \mathbf{L}^{2}(\Omega)\right)$ with $\mathbf{v}(\cdot, T)=\mathbf{0}$,

$$
\begin{gathered}
\int_{0}^{T}\left\langle\partial_{t} c, \eta\right\rangle \mathrm{d} t+\int_{0}^{T}(\nabla w, \nabla \eta) \mathrm{d} t=\int_{0}^{T} \mathbf{a}(c, \eta, \mathbf{u}) \mathrm{d} t \\
\int_{0}^{T}(w, \eta) \mathrm{d} t=\gamma^{2} \int_{0}^{T}(\nabla c, \nabla \eta) \mathrm{d} t+\int_{0}^{T}\left(\Phi^{\prime}(c), \eta\right) \mathrm{d} t \\
-\int_{0}^{T}\left(\mathbf{u}, \partial_{t} \mathbf{v}\right) \mathrm{d} t+\int_{0}^{T}(\nabla \mathbf{u}: \nabla \mathbf{v}) \mathrm{d} t+\int_{0}^{T} B(\mathbf{u}, \mathbf{u}, \mathbf{v}) \mathrm{d} t+\int_{0}^{T} \mathbf{a}(c, w, \mathbf{v}) \mathrm{d} t=\left(\mathbf{u}_{0}(\cdot), \mathbf{v}(\cdot, 0)\right)
\end{gathered}
$$

Proof. For any $\eta \in L^{2}\left(0, T ; W^{1,4}(\Omega)\right) \cap H^{1}\left(0, T ; L^{2}(\Omega)\right)$ there exists a subsequence $\eta_{h, \tau}(t) \in S^{h}$ such that

$$
\eta_{h, \tau} \rightarrow \eta \quad \text { strongly in } L^{2}\left(0, T ; H^{1}(\Omega)\right) .
$$

We choose $\chi \equiv \eta_{h, \tau}$ in 4.21a and now analyse the subsequent terms. Firstly, 2.18 and 4.24 imply that as $h \rightarrow 0$,

$$
\begin{aligned}
& \left|\int_{0}^{T}\left[\left(\partial_{t} c_{h, \tau}, \eta_{h, \tau}\right)_{h}-\left(\partial_{t} c_{h, \tau}, \eta_{h, \tau}\right)\right] \mathrm{d} t\right| \\
& =\mid-\int_{0}^{T}\left(c_{h, \tau}, \partial_{t} \eta_{h, \tau}\right)_{h} \mathrm{~d} t+\left(c_{h, \tau}(\cdot, T), \eta_{h, \tau}(\cdot, T)\right)_{h}-\left(c_{h, \tau}(\cdot, 0), \eta_{h, \tau}(\cdot, 0)\right)_{h} \\
& \quad+\int_{0}^{T}\left(c_{h, \tau}, \partial_{t} \eta_{h, \tau}\right) \mathrm{d} t-\left(c_{h, \tau}(\cdot, T), \eta_{h, \tau}(\cdot, T)\right)+\left(c_{h, \tau}(\cdot, 0), \eta_{h, \tau}(\cdot, 0)\right) \mid \rightarrow 0 .
\end{aligned}
$$

Furthermore, it follows from 2.5 , 4.23c and 4.34 that

$$
\int_{0}^{T}\left(\partial_{t} c_{h, \tau}, \eta_{h, \tau}\right) \mathrm{d} t \rightarrow \int_{0}^{T}\left\langle\partial_{t} c, \eta\right\rangle \mathrm{d} t \quad \text { as } h \rightarrow 0 .
$$

Combining (4.35) and (4.36) shows that

$$
\int_{0}^{T}\left(\partial_{t} c_{h, \tau}, \eta_{h, \tau}\right)_{h} \mathrm{~d} t \rightarrow \int_{0}^{T}\left\langle\partial_{t} c, \eta\right\rangle \mathrm{d} t \quad \text { as } h \rightarrow 0
$$

In view of (4.34) and (4.23d) it follows that as $h \rightarrow 0$,

$$
\int_{0}^{T}\left(\nabla w_{h, \tau}^{+}, \nabla \eta_{h, \tau}\right) \mathrm{d} t \rightarrow \int_{0}^{T}(\nabla w, \nabla \eta) \mathrm{d} t
$$

From Lemma 4.5, 4.23b, 4.26 and 4.34) we conclude that as $h \rightarrow 0$,

$$
\int_{0}^{T} \mathbf{a}\left(c_{h, \tau}^{-}, \eta_{h, \tau}, \mathbf{u}_{h, \tau}^{-}\right) \mathrm{d} t \rightarrow \int_{0}^{T} \mathbf{a}(c, \eta, \mathbf{u}) \mathrm{d} t .
$$

Combining (4.37), 4.38) and 4.39) yields 4.33a, on recalling 4.22). 
From (2.18), 4.24, 4.23a, 4.23d) and 4.34) we infer that as $h \rightarrow 0$,

$$
\int_{0}^{T}\left[\gamma^{2}\left(\nabla c_{h, \tau}^{+}, \nabla \eta_{h, \tau}\right)+\left(c_{h, \tau}^{-}-w_{h, \tau}^{+}, \eta_{h, \tau}\right)_{h}\right] \mathrm{d} t \rightarrow \int_{0}^{T}\left[\gamma^{2}(\nabla c, \nabla \eta)+(c-w, \eta)\right] \mathrm{d} t .
$$

Furthermore, setting $F\left(c, c_{h, \tau}\right)=c_{h, \tau}^{2}+c^{2}+c_{h, \tau} c$ and recalling 4.22 we have

$$
\begin{aligned}
\left|\int_{0}^{T}\left[\left(\phi\left(c_{h, \tau}^{+}\right), \eta_{h, \tau}\right)-(\phi(c), \eta)\right] \mathrm{d} t\right| \\
\quad \leqslant\left|\int_{0}^{T}\left(\phi\left(c_{h, \tau}^{+}\right)-\phi(c), \eta\right) \mathrm{d} t\right|+\left|\int_{0}^{T}\left(\phi\left(c_{h, \tau}^{+}\right), \eta_{h, \tau}-\eta\right) \mathrm{d} t\right| \\
\quad \leqslant \int_{0}^{T}\|\eta\|_{0,4}\left\|F\left(c, c_{h, \tau}\right)\right\|_{0,4}\left\|c_{h, \tau}^{+}-c\right\| \mathrm{d} t+\int_{0}^{T}\left\|\phi\left(c_{h, \tau}^{+}\right)\right\|\left\|\eta_{h, \tau}-\eta\right\| \mathrm{d} t \\
\quad \leqslant C \int_{0}^{T}\|\eta\|_{0,4}\left\|c_{h, \tau}\right\|_{0,8}^{4}\left\|c_{h, \tau}^{+}-c\right\| \mathrm{d} t+C \int_{0}^{T}\left\|c_{h, \tau}^{+}\right\|_{0,6}^{3}\left\|\eta_{h, \tau}-\eta\right\| \mathrm{d} t \\
\quad \leqslant C \int_{0}^{T}\|\eta\|_{1}\left\|c_{h, \tau}\right\|_{1}^{4}\left\|c_{h, \tau}^{+}-c\right\| \mathrm{d} t+C \int_{0}^{T}\left\|c_{h, \tau}^{+}\right\|_{1}^{3}\left\|\eta_{h, \tau}-\eta\right\| \mathrm{d} t .
\end{aligned}
$$

From 4.18, 4.24, 4.41, 4.23b and 4.34) we have

$$
\int_{0}^{T}\left(\phi\left(c_{h, \tau}^{+}\right), \eta_{h, \tau}\right)_{h} \mathrm{~d} t \rightarrow \int_{0}^{T}(\phi(c), \eta) \mathrm{d} t \quad \text { as } h \rightarrow 0 .
$$

Combining (4.40) and (4.42) yields (4.33b), on recalling (4.22).

For $\mathbf{v} \in L^{2}\left(0, T ; \mathbf{W}_{0}\right) \cap H^{1}\left(0, T ; \mathbf{L}^{2}(\Omega)\right)$ there exists $\mathbf{v}_{h, \tau}(t) \in \mathbf{W}_{0}^{h}$ such that

$$
\begin{array}{ll}
\mathbf{v}_{h, \tau} \rightarrow \mathbf{v} & \text { strongly in } L^{2}\left(0, T ; \mathbf{H}^{1}(\Omega)\right), \\
\partial_{t} \mathbf{v}_{h, \tau} \rightarrow \partial_{t} \mathbf{v} & \text { weakly in } L^{2}\left(0, T ; \mathbf{L}^{2}(\Omega)\right) .
\end{array}
$$

We choose $\chi \equiv \mathbf{v}_{h, \tau}$ in $4.21 \mathrm{c}$ ) and now analyse the subsequent terms. Since $\mathbf{v}_{h, \tau}(\cdot, T)=\mathbf{0}$ we have

$$
\int_{0}^{T}\left(\partial_{t} \mathbf{u}_{h, \tau}, \mathbf{v}_{h, \tau}\right) \mathrm{d} t=-\int_{0}^{T}\left(\mathbf{u}_{h, \tau}, \partial_{t} \mathbf{v}_{h, \tau}\right) \mathrm{d} t-\left(\mathbf{u}_{h, \tau}(\cdot, 0), \mathbf{v}_{h, \tau}(\cdot, 0)\right)
$$

and from 4.45, 4.26, and 4.44, we have

$$
\int_{0}^{T}\left(\partial_{t} \mathbf{u}_{h, \tau}, \mathbf{v}_{h, \tau}\right) \mathrm{d} t \rightarrow-\int_{0}^{T}\left(\mathbf{u}, \partial_{t} \mathbf{v}\right) \mathrm{d} t-\left(\mathbf{u}_{0}(\cdot), \mathbf{v}(\cdot, 0)\right) \quad \text { as } h \rightarrow 0 .
$$

Using arguments similar to the proof of Lemma 4.5 and noting $4.23 \mathrm{e},(4.26)$ and $(4.43)$ it follows that as $h \rightarrow 0$,

$$
\int_{0}^{T} B\left(\mathbf{u}_{h, \tau}^{-}, \mathbf{u}_{h, \tau}^{+}, \mathbf{v}_{h, \tau}\right) \mathrm{d} t \rightarrow \int_{0}^{T} B(\mathbf{u}, \mathbf{u}, \mathbf{v}) \mathrm{d} t,
$$


and from 4.23e and 4.43 we find that as $h \rightarrow 0$,

$$
\int_{0}^{T}\left(\nabla \mathbf{u}_{h, \tau}^{+}: \nabla \mathbf{v}_{h, \tau}\right) \mathrm{d} t \rightarrow \int_{0}^{T}(\nabla \mathbf{u}: \nabla \mathbf{v}) \mathrm{d} t .
$$

From Lemma 4.5, 4.23b, 4.23d and 4.43 it follows that as $h \rightarrow 0$,

$$
\int_{0}^{T} \mathbf{a}\left(c_{h, \tau}^{-}, w_{h, \tau}^{+}, \mathbf{v}\right) \mathrm{d} t \rightarrow \int_{0}^{T} \mathbf{a}(c, w, \mathbf{v}) \mathrm{d} t .
$$

Combining 4.46-4.49) yields 4.33c on recalling 4.22.

REMARK 4.3 We note that there is an inconsistency of notation between this section and Section 3 . since in this section $\{c, w, \mathbf{u}\}$ is used to denote the solution of $4.33 \mathrm{a}-\mathrm{c})$, whereas in Section 3 . $\{c, w, \mathbf{u}\}$ is used to denote the solution of the smoother problem $(\mathbf{P})$.

\section{Numerical results}

In practice the concentration, $c$, rapidly varies over the interfacial regions between the two fluids, while away from the interface, in the bulk regions, it is close to \pm 1 . This interfacial region is of thickness $O(\gamma \ln \gamma)$ and to effectively model it we require an adaptive mesh that is locally refined close to and inside of the interfacial region and coarsened elsewhere. We now define our mesh refinement strategy: if

Given an initial mesh $\mathcal{T}^{0}$, for time step $n$ an element, $\kappa$, with vertices $\mathbf{x}_{\kappa, i}, i=1,2,3$, is refined

$$
\max _{i}\left|c_{h}^{n-1}\left(\mathbf{x}_{\kappa, i}\right)\right|<1-\delta
$$

and $h_{\kappa}>\gamma / 2$ where $\delta=O(\gamma)$; in practice this guarantees that there are at least eight grid points in the interfacial region. This element is repeatedly refined, using uniform refinement, until $h_{\kappa}<\gamma / 2$. Elements are coarsened if $\kappa$ and its neighbouring elements $\Omega_{\kappa}=\left\{K \in \mathcal{T}^{n-1}: \bar{\kappa} \cap \bar{K} \neq \emptyset\right\}$, with the set of vertices $\omega_{\kappa}$, are such that

$$
\max _{\mathbf{x} \in \omega_{\kappa}}\left|c_{h}^{n-1}(\mathbf{x})\right|>1-\delta
$$

We call this grid $\mathcal{T}^{n}$ and use it for the chemical potential, $w$, as well. The space of continuous piecewise linear functions associated with this space is denoted by $S^{n, c}$ and we denote the dimension of $S^{n, c}$ by $N^{n, c}$. We use standard continuous piecewise linear interpolation to transfer functions in $S^{n-1, c}$ to $S^{n, c}$.

For the velocity the divergence free space, $\mathbf{W}_{0}^{h}$, is not constructed and the pressure function, $p$, remains in the system. We use a fixed uniform mesh, $\mathcal{T}^{h}$, for this problem and the mixed finite element $\left(S^{h / 2}\right)^{2} \times S^{h}$; this space, (P1 iso P2 - P1), is known to be inf-sup stable (see [13]). Note that the space $S^{h / 2}$ need not be related to the concentration mesh and in general is not. We denote the dimensions of $S^{h / 2}$ and $S^{h}$ by $N^{h, u}$ and $N^{h, p}$, respectively.

We obtain the following fully discrete approximation of $(2.11 \mathrm{a}-\mathrm{f})$ : 
$\left(\tilde{\mathbf{P}}_{h, \tau}\right)$ For $n \geqslant 1$ find $\left\{c_{h}^{n}, w_{h}^{n}, \mathbf{u}_{h}^{n}, p_{h}^{n}\right\} \in S^{n, c} \times S^{n, c} \times\left(S^{h / 2}\right)^{2} \times S^{h}$ such that

$$
\begin{aligned}
& \left(\delta_{\tau} c_{h}^{n}, \chi\right)_{h}+\frac{1}{\mathrm{Pe}}\left(b\left(c_{h}^{n-1}\right) \nabla w_{h}^{n}, \nabla \chi\right)=\mathbf{a}\left(c_{h}^{n-1}, \chi, \mathbf{u}_{h}^{n-1}\right) \quad \forall \chi \in S^{n, c}, \\
& \gamma^{2}\left(\nabla c_{h}^{n}, \nabla \chi\right)=\left(w_{h}^{n}-\phi\left(c_{h}^{n}\right)+c_{h}^{n-1}, \chi\right)_{h} \quad \forall \chi \in S^{n, c}, \\
& \left(\delta_{\tau} \mathbf{u}_{h}^{n}, \boldsymbol{\chi}\right)+\frac{1}{\operatorname{Re}}\left(\nabla \mathbf{u}_{h}^{n}: \nabla \chi\right)+B\left(\mathbf{u}_{h}^{n-1}, \mathbf{u}_{h}^{n}, \boldsymbol{\chi}\right)+K \mathbf{a}\left(c_{h}^{n-1}, w_{h}^{n}, \chi\right) \\
& =\left(p_{h}^{n}, \nabla \cdot \chi\right) \quad \forall \chi \in\left(S^{h / 2}\right)^{2}, \\
& \left(\nabla \cdot \mathbf{u}_{h}^{n}, q_{h}\right)=0 \quad \forall q_{h} \in S^{h}, \\
& c_{h}^{0}(x)=Q^{h}\left(c_{0}(x)\right), \quad \mathbf{u}_{h}^{0}(x)=\mathbf{P}^{h}\left(\mathbf{u}_{0}(x)\right) \\
& \forall x \in \Omega \text {, } \\
& \text { on } \partial \Omega \times(0, T) \text {, }
\end{aligned}
$$

where $b(c)=\exp \left(-c^{2}\right)$ and $\mathbf{g} \cdot \mathbf{n}=0$ on $\partial \Omega$

We now discuss algorithms for solving the resulting system of algebraic equations for $\left\{c_{h}^{n}, w_{h}^{n}, \mathbf{u}_{h}^{n}, p_{h}^{n}\right\}$ arising at each time level from the approximation $\left(\tilde{\mathbf{P}}_{h, \tau}\right)$. We note that with this approximation at each time level we are required to solve a Cahn-Hilliard equation followed by a Navier-Stokes equation.

Let $\mathbf{C}^{n}=\left\{c_{1}^{n}, c_{2}^{n}, \ldots, c_{N^{n, c}}^{n}\right\}$ be the vector of coefficients for $c_{h}^{n}$ with respect to the standard basis $S^{n, c}=\left\langle\psi_{i}^{n, c}\right\rangle_{1}^{N^{n, c}}$ and similarly for $\mathbf{W}^{n}, \mathbf{U}^{n}$ and $\mathbf{P}^{n}$. Then the system 5.1a-f) can be rewritten as:

Find $\mathbf{C}^{n}, \mathbf{W}^{n} \in \mathbb{R}^{N^{n, c}}, \mathbf{U}^{n} \in\left(\mathbb{R}^{N^{h, u}}\right)^{2}$ and $\mathbf{P}^{n} \in \mathbb{R}^{N^{h, p}}$ such that

$$
\begin{gathered}
\widehat{M}^{n, c} \mathbf{C}^{n}+\frac{\tau_{n}}{\mathrm{Pe}} D^{n, c} \mathbf{W}^{n}=\widehat{M}^{n, c} \mathbf{C}^{n-1}+\tau_{n} \mathbf{F}\left(c_{h}^{n-1}, \mathbf{u}_{h}^{n-1}\right), \\
-\widehat{M}^{n, c} \mathbf{W}^{n}+\widehat{M}^{n, c} \phi\left(\mathbf{C}^{n}\right)+\gamma^{2} A^{n, c} \mathbf{C}^{n}=\widehat{M}^{n, c} \mathbf{C}^{n-1}, \\
\left(\mathbf{M}^{h, u}+\frac{\tau_{n}}{\operatorname{Re}} \mathbf{A}^{h, u}+\tau_{n} \mathbf{S}^{n-1, h, u}\right) \mathbf{U}^{n}-\tau_{n}\left(B^{h}\right)^{T} \mathbf{P}^{n}=\mathbf{M}^{h, u} \mathbf{U}^{n-1}-\tau_{n} K \mathbf{G}\left(c_{h}^{n-1}, w_{h}^{n}\right), \\
B^{h} \mathbf{U}^{n}=\mathbf{0},
\end{gathered}
$$

where the Cahn-Hilliard matrices and right hand side are given by

$$
\begin{aligned}
A^{n, c}(i, j) & :=\int_{\Omega} \nabla \psi_{i}^{n, c} \cdot \nabla \psi_{j}^{n, c} \mathrm{~d} x, & D^{n, c}(i, j) & :=\int_{\Omega} b\left(\mathbf{c}_{h}^{n-1}\right) \nabla \psi_{i}^{n, c} \cdot \nabla \psi_{j}^{n, c} \mathrm{~d} x, \\
\widehat{M}^{n, c}(i, i) & :=\int_{\Omega} \pi^{h}\left[\left(\psi_{i}^{n, c}\right)^{2}\right] \mathrm{d} x, & \mathbf{F}_{i}\left(c_{h}^{n-1}, \mathbf{u}_{h}^{n-1}\right) & :=\int_{\Omega} c_{h}^{n-1} \nabla \psi_{i}^{n, c} \cdot \mathbf{u}_{h}^{n-1} \mathrm{~d} x,
\end{aligned}
$$

and the Navier-Stokes matrices are given by

$$
\begin{gathered}
\mathbf{A}^{h, u}=\left[\begin{array}{cc}
A^{h, u} & 0 \\
0 & A^{h, u}
\end{array}\right], \quad \mathbf{M}^{h, u}=\left[\begin{array}{cc}
M^{h, u} & 0 \\
0 & M^{h, u}
\end{array}\right], \quad \mathbf{S}^{n-1, h, u}=\left[\begin{array}{cc}
S^{n-1, h, u} & 0 \\
0 & S^{n-1, h, u}
\end{array}\right], \\
B^{h}=\left[B_{1}^{h}, B_{2}^{h}\right], \quad \mathbf{G}=\left[\begin{array}{l}
\mathbf{G}^{1}\left(c_{h}^{n-1}, w_{h}^{n}\right) \\
\mathbf{G}^{2}\left(c_{h}^{n-1}, w_{h}^{n}\right)
\end{array}\right]
\end{gathered}
$$


with

$$
\begin{gathered}
A^{h, u}(i, j):=\int_{\Omega} \nabla \psi_{i}^{h, u} \cdot \nabla \psi_{j}^{h, u} d x, \quad M^{h, u}(i, j):=\int_{\Omega} \psi_{i}^{h, u} \psi_{j}^{h, u} \mathrm{~d} x, \\
S^{n-1, h, u}:=\frac{1}{2} \int_{\Omega}\left[\mathbf{u}^{h, n-1} \cdot \nabla \psi_{i}^{h, u}\right] \cdot \psi_{j}^{h, u} \mathrm{~d} x-\frac{1}{2} \int_{\Omega}\left[\mathbf{u}^{h, n-1} \cdot \nabla \psi_{j}^{h, u}\right] \cdot \psi_{i}^{h, u} \mathrm{~d} x, \\
B_{1}^{h, u}(i, j):=\int_{\Omega} \frac{\partial \psi_{i}^{h, u}}{\partial x_{1}} \psi_{j}^{h, p} \mathrm{~d} x, \quad B_{2}^{h, u}(i, j):=\int_{\Omega} \frac{\partial \psi_{i}^{h, u}}{\partial x_{2}} \psi_{j}^{h, p} \mathrm{~d} x, \\
\mathbf{G}_{i}^{1}\left(c_{h}^{n-1}, w_{h}^{n}\right):=\int_{\Omega} c_{h}^{n-1} \frac{\partial w_{h}^{n}}{\partial x_{1}} \psi_{i}^{h, u} \mathrm{~d} x, \quad \mathbf{G}_{i}^{2}\left(c_{h}^{n-1}, w_{h}^{n}\right):=\int_{\Omega} c_{h}^{n-1} \frac{\partial w_{h}^{n}}{\partial x_{2}} w_{h}^{n} \psi_{i}^{h, u} \mathrm{~d} x .
\end{gathered}
$$

Here $\left\langle\psi_{i}^{n, c}\right\rangle_{1}^{N^{n, c}},\left\langle\psi_{i}^{h, u}\right\rangle_{1}^{N^{h, u}}$ and $\left\langle\psi_{i}^{h, p}\right\rangle_{1}^{N^{h, p}}$ are the standard basis functions for $S^{n, c}, S^{h / 2}$ and $S^{h}$ respectively.

To solve the Cahn-Hilliard equation we use the multigrid solver given in [14] which produces an efficient and reliable method with optimal convergence. For the Navier-Stokes equation, we have an Oseen type equation, which is solved using preconditioned GMRES (see [31]), where the $F_{p}$ preconditioner is used (see [24]). This solver is known to be optimal with respect to mesh refinement and has mild dependence on the Reynolds number. For further details and numerical examples showing the efficiency of this solution method see [25].

We display two numerical experiments; the first shows the evolution of a cross-shaped interface to a circle and the second is similar to the lid driven cavity experiment seen in [8].

\subsection{Rotational boundary condition: cross to circle}
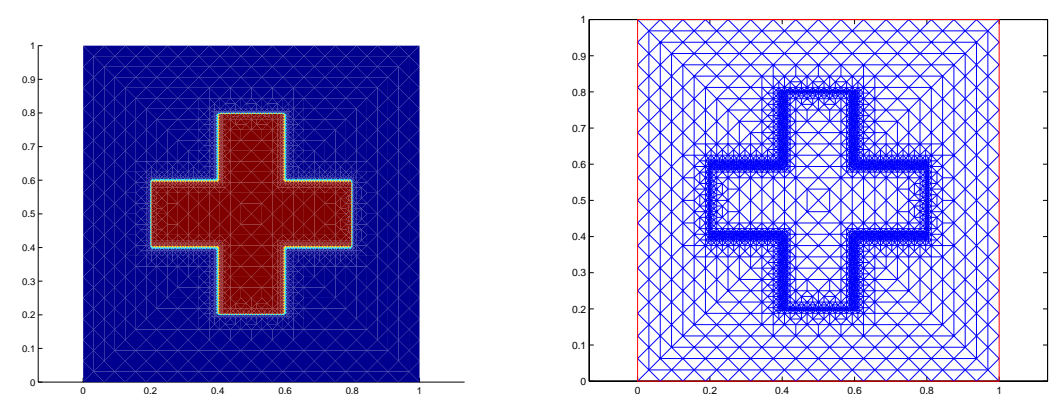

FIG. 1. Initial data for the concentration $c_{h, \tau}$ and the initial mesh.

In this experiment we take $\Omega=(0,1)^{2}$ and as initial data for the concentration we take a cross set inside $\Omega$ (see Figure 11; for the velocity we take $\mathbf{u}_{0}=\left[x_{2},-x_{1}\right]$ and $\mathbf{g}=\left[x_{2},-x_{1}\right]$. We set $v=1 / 100, \mathrm{Pe}=1000, K=0.001$ and $\gamma=1 / 120$ and we use a fixed time step with $\tau=0.05$. For the velocity space we use a uniform mesh of size $h_{\mathbf{u}} \approx 1 / 90$.

Figures 24 show the evolution of the concentration for this problem together with the corresponding meshes. 

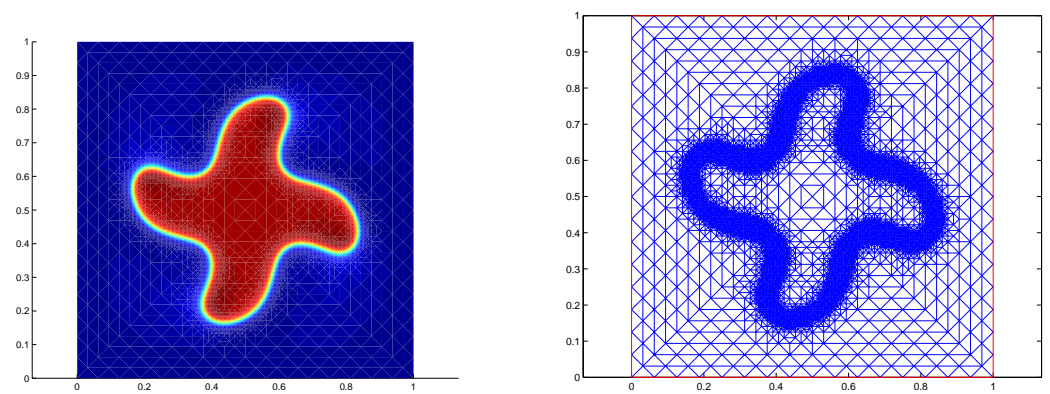

FIG. 2. Concentration $c_{h, \tau}$ and mesh at $t=3$.
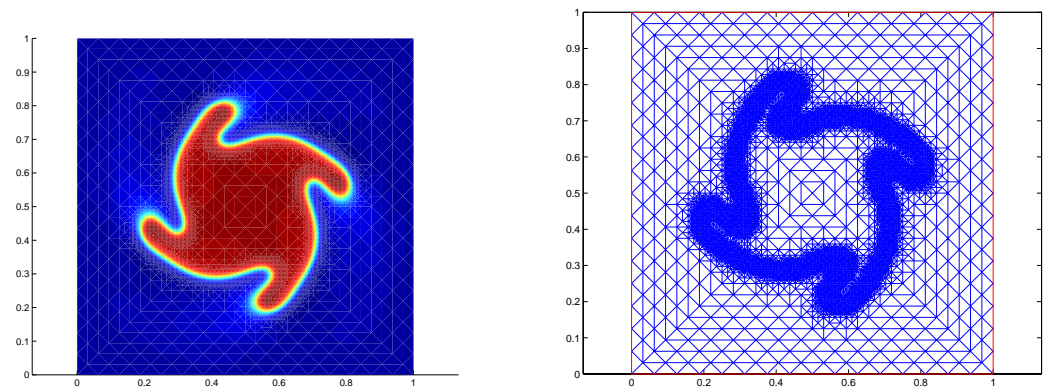

FIG. 3. Concentration $c_{h, \tau}$ and mesh at $t=7$.
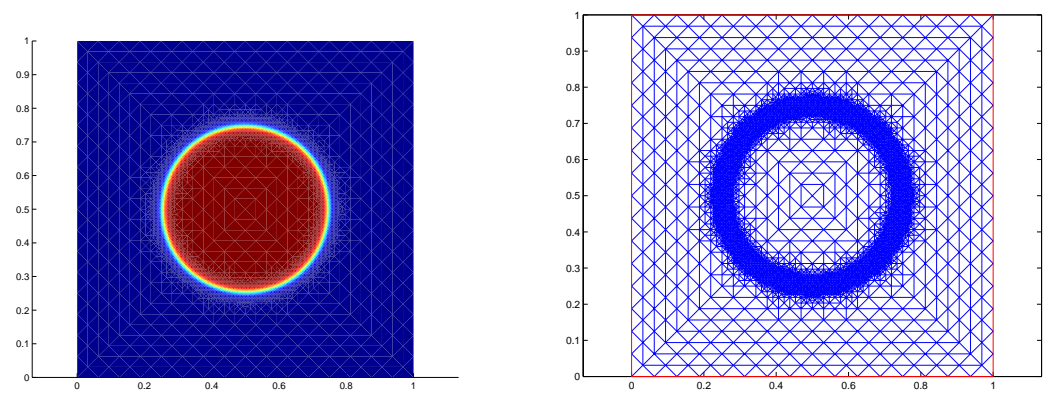

FIG. 4. Concentration $c_{h, \tau}$ and mesh at $t=20$. 


\subsection{Lid driven cavity boundary condition}

In this experiment we take $\Omega=(0,1)^{2}$ and as initial data for the concentration we take the horizontal line $x_{2}=0.5$. For the velocity we take the initial data to be the Stokes solution to the lid driven cavity problem and for boundary data we take $\mathbf{g}=\left[x_{1}^{2}\left(1-x_{1}\right)^{2}, 0\right]$ for $x_{2}=1$, and zero otherwise. We set $v=1 / 500, \mathrm{Pe}=200, K=1 / 5000$ and $\gamma=1 / 120$ and we use a fixed time step with $\tau=0.025$. The velocity space is a uniform mesh of size $h_{\mathbf{u}} \approx 1 / 90$.

The evolution of the concentration for this problem is shown through Figures $5+8$ (see [8] for a similar problem). The corresponding adapted meshes are also given. Figures 9 10 show contour plots of the concentration and the velocity field. From the magnified images we can see the velocity at the front of the fluid is almost perpendicular to the interface and thus forces it to move, while behind this front the velocity is almost tangential and hence in this region the movement of the interface is small.

We conclude with Figure 11 in which we see the flow at time $t=20$ together with a contour plot of the concentration.
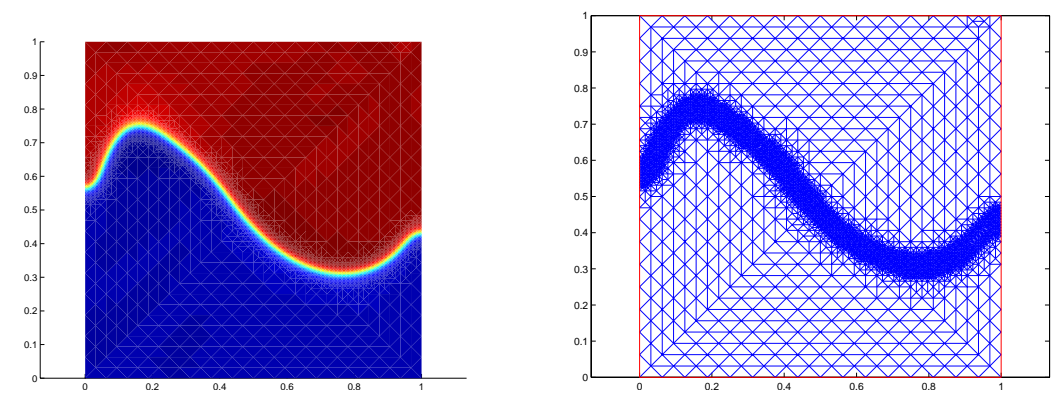

FIG. 5. Concentration $c_{h, \tau}$ and mesh at $t=3.4$.
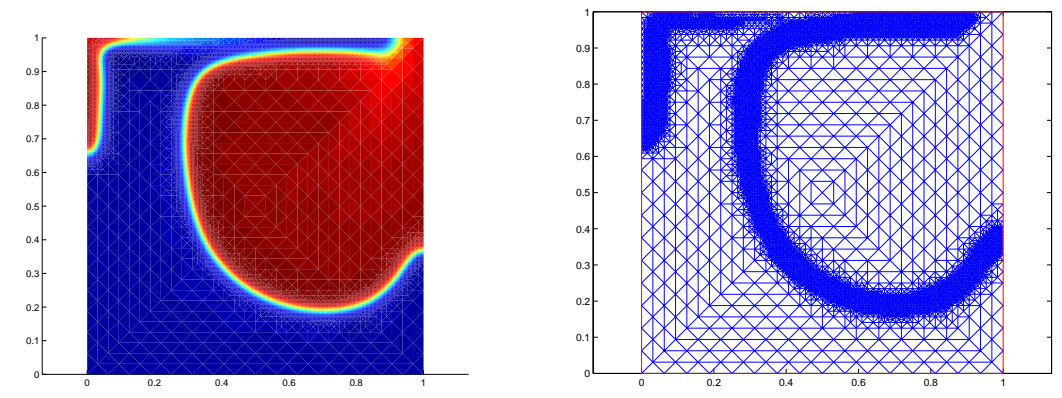

FIG. 6. Concentration $c_{h, \tau}$ and mesh at $t=6.5$. 

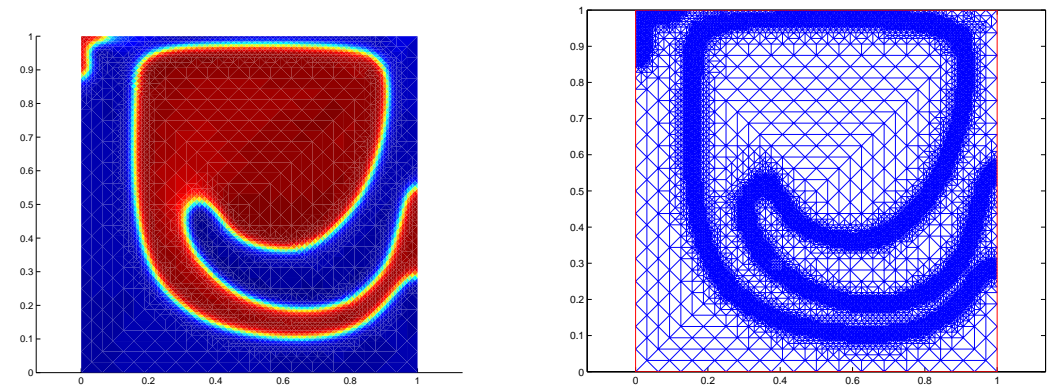

FIG. 7. Concentration $c_{h, \tau}$ and mesh at $t=12.5$.
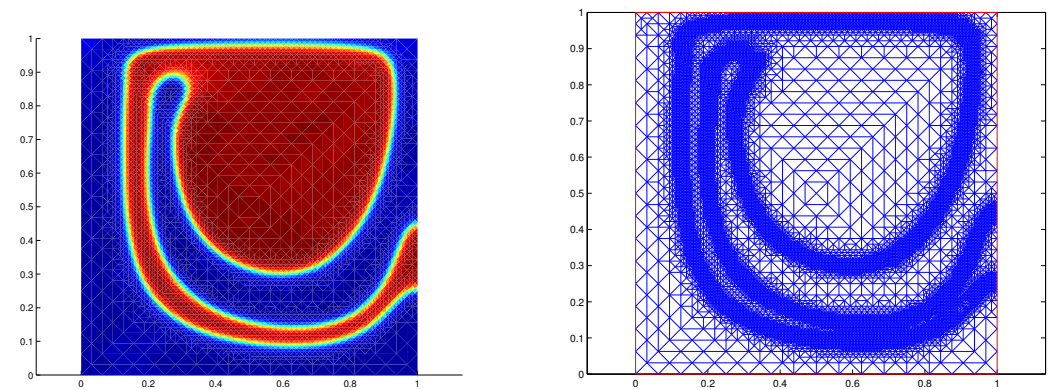

FIG. 8. Concentration $c_{h, \tau}$ and mesh at $t=20$.

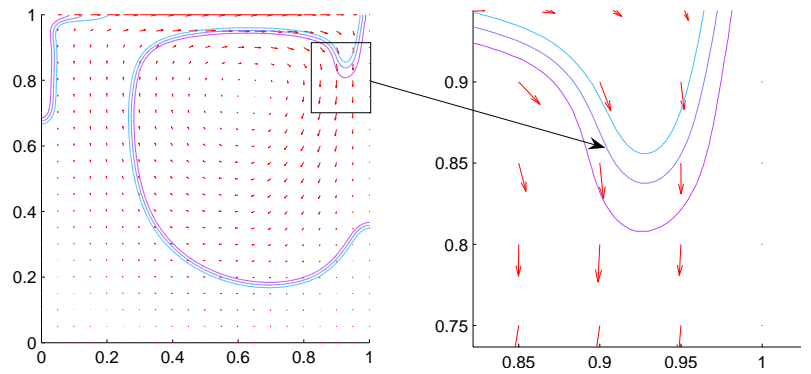

FIG. 9. Flow and concentration at $t=7$. 


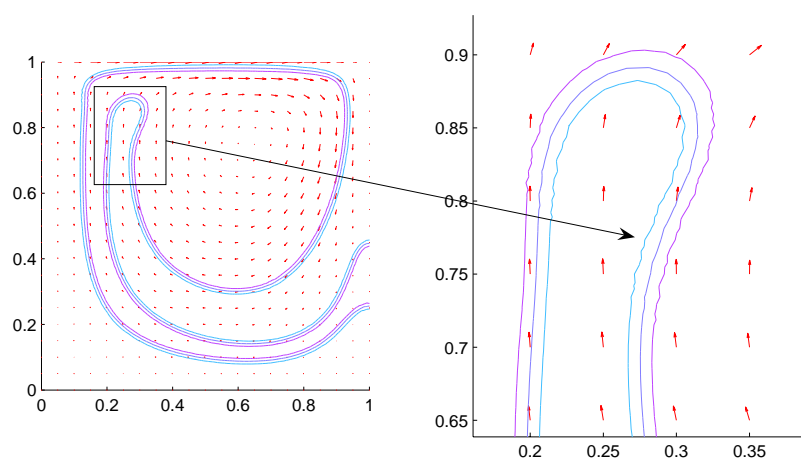

FIG. 10. Flow and concentration at $t=16.5$.

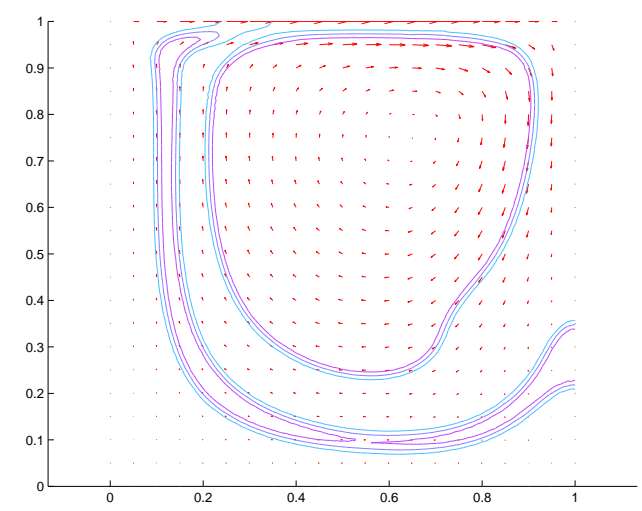

FIG. 11. Flow and concentration at $t=20$.

\section{Acknowledgements}

The authors would like to thank Charlie Elliott for his valuable insights and observations during the writing of this paper.

\section{REFERENCES}

1. Anderson, D. M., McFadden, G. B., \& Wheeler, A. A. Diffuse interface methods in fluid mechanics. Ann. Rev. Fluid Mech. 30 (1998), 139-165. MR 1609626

2. Badalassi, V. E., Ceniceros, H. D., \& Banerjee, S. Computation of multiphase systems with phase field models. J. Comput. Phys. 190 (2003), 371-397. Zbl 1076.76517 MR 2013023

3. Barrett, J. W., \& Blowey, J. F. An error bound for the finite element approximation of the Cahn-Hilliard equation with logarithmic free energy. Numer. Math. 72 (1995), 1-20. Zbl 0851.65070 MR 1359705 
4. Barrett, J. W., \& Blowey, J. F. Finite element approximation of the Cahn-Hilliard equation with concentration mobility. Math. Comp. 68 (1999), 487-517. Zbl pre01267989 MR 1609678

5. Barrett, J. W., Langdon, S., \& Nuernberg, R. Finite element approximation of a sixth order nonlinear degenerate parabolic equation. Numer. Math. 96 (2004), 401-434. Zbl 1041.65076 MR 2028722

6. Blowey, J. F., Copetti, M. I. M., \& Elliott, C. M. Numerical analysis of a model for phase separation of a multicomponent alloy. IMA J. Numer. Anal. 16 (1996), 111-139. Zbl 0857.65137 MR 1367400

7. BOYER, F. Mathematical study of multiphase flow under shear through order parameter formulation. Asymptotic Anal. 20 (1999), 175-212. Zbl 0937.35123 MR 1700669

8. BoYER, F. A theoretical and numerical model for the study of incompressible mixture flows. Comput. Fluids 31 (2002), 41-68. Zbl 1057.76060

9. Boyer, F., Chupin, L., \& FrAnCK, B. A. Numerical study of viscoelastic mixtures through a CahnHilliard fluid. Eur. J. Mech. B Fluids 23 (2004), 759-780. Zbl 1058.76531 MR 2077449

10. CAhn, J. W. On spinodal decomposition. Acta Metall. Mater. 9 (1961), 795-801.

11. CAHn, J. W., \& Hilliard, J. E. Free energy of a nonuniform system, I, interfacial free energy. J. Chem. Phys. 28 (1958), 258-267.

12. Chupin, L. Existence result for a mixture of non-newtonian flows with stress diffusion using the Cahn-Hilliard formulation. Discrete Contin. Dynam. Systems Ser. B 3 (2003), 45-68. Zbl pre01894896 MR 1951567

13. Ciarlet, P., JR., \& Girault, V. Condition inf-sup pour l'élément fini de Taylor-Hood $P_{2}$-iso$P_{1}$, 3-D; application aux équations de Maxwell. C. R. Math. Acad. Sci. Paris 335 (2002), 827-832. Zbl 1021.78009 MR 1947708

14. KAY, D., \& WELFORD, R. A multigrid finite element solver for the Cahn-Hilliard equation. J. Comput. Phys. 212 (2006), 288-304. Zbl 1081.65091 MR 2183612

15. Elliott, C. M. The Cahn-Hilliard model for the kinetics of phase separation. Mathematical Model for Phase Change Problems (Óbidos, 1988), Int. Ser. Numer. Math. 88, Birkhäuser, Basel (1989), 35-73. Zbl 0692.73003 MR 1038064

16. Elliott, C. M., French, D. A., \& Milner, F. A. A second order splitting method for the CahnHilliard equation. Numer. Math. 54 (1989), 575-590. Zbl 0668.65097 MR 0978609

17. Elliott, C. M., Kay, D., \& Styles, V. k Finite element analysis of a current density-electric field formulation of bean's model for superconductivity. IMA J. Numer. Anal. 25 (2005), 182-204. Zbl 1100.78018 MR 2110240

18. Elliott, C. M., \& Stuart, A. M. The global dynamics of discrete semilinear parabolic equations. SIAM J. Numer. Anal. 30 (1993), 1622-1663. Zbl 0792.65066 MR 1249036

19. FENG, X. Fully discrete finite element approximation of the Navier-Stokes-Cahn-Hilliard diffuse interface model for two-phase flows. SIAM J. Numer. Anal. 44 (2006), 1049-1072. Zbl pre05167765 MR 2231855

20. Feng, X., \& Prohl, A. Numerical analysis of the Cahn-Hilliard equation and approximation of the Hele-Shaw problem. Interfaces Free Bound. 7 (2005), 1-28. Zbl 1072.35150 MR 2126141

21. Girault, V., \& Raviart, P. A. Finite Element Methods for the Navier-Stokes Equations: Theory and Algorithms. Springer Ser. Comput. Math., Springer, New York, 1986. Zbl 0585.65077

22. Hohenberg, P. C., \& Halperin, B. I. Theory of dynamic critical phenomena. Rev. Mod. Phys. 49 (1977), 435-479.

23. JACQMin, D. Calculations of two-phase Navier-Stokes flows using phase-field modelling. J. Comput. Phys. 155 (1999), 96-127. Zbl 0966.76060 MR 1716497

24. KAY, D., Loghin, D., \& WATHEN, A. A preconditioner for the steady-state Navier-Stokes equations. SIAM J. Sci. Comput. 24 (2002), 237-256. Zbl 1013.65039 MR 1924423 
25. KAY, D., \& WELFORD, R. Efficient numerical solution of Cahn-Hilliard-Navier-Stokes fluids in 2D. SIAM J. Sci. Comput., to appear.

26. KIM, J. A diffuse-interface model for axisymmetric immiscible two-phase flow. Appl. Math. Comput. 160 (2005), 589-606. Zbl pre02132586 MR 2102831

27. Kim, J., Kang, K., \& Lowengrub, J. Conservative multigrid methods for Cahn-Hilliard fluids. J. Comput. Phys. 193 (2004), 511-543. Zbl 1109.76348 MR 2030475

28. Lions, J.-L. On some questions in boundary value problems of mathematical physics. Contemporary Development in Continuum Mechanics and Partial Differential Equations (Rio de Janeiro, 1977), NorthHolland Math. Stud. 30, North-Holland (1978), 284-346. Zbl 0404.35002 MR 0519648

29. Lions, J.-L., \& Magenes, E. Non-Homogeneous Boundary Value Problems and Applications. Volume I, Springer, Berlin (1972). Zbl 0223.35039 MR 0350177

30. Rodrigues, J.-F. Obstacle Problems in Mathematical Physics. North-Holland Math. Stud. 134, NorthHolland, Amsterdam (1987). Zbl 0606.73017 MR 0880369

31. SAAD, Y., \& Schultz, M. H. GMRES: A generalized minimum residual algorithm for solving nonsymmetric linear systems. SIAM J. Sci. Statist. Comput. 7 (1986), 856-869. Zbl 0599.65018 MR 0848568 